\title{
Interacting Information Cascades: On the Movement of Conventions Between Groups
}

\author{
James C.D. Fisher* John Wooders ${ }^{\dagger}$
}

January 16, 2015

\begin{abstract}
When a decision maker is a member of multiple social groups, her actions may cause information to "spill over" from one group to another. We study the nature of these spillovers in an observational learning game where two groups interact via a common player, and where conventions emerge when players follow the decisions of the members of their own groups rather than their own private information. We show that: (i) if a convention develops in one group but not the other group, then the convention spills over via the common player; (ii) when conventions disagree, then the common player's decision breaks the convention in one group; and (iii) when no conventions have developed, then the common player's decision triggers conventions on the same action in both groups. We also find that information spillovers may reduce welfare.
\end{abstract}

${ }^{*}$ Department of Economics, University of Arizona, fisherjc@email.arizona.edu.

${ }^{\dagger}$ Economics Discipline Group, University of Technology Sydney, john.wooders@uts.edu.au. 


\section{Introduction}

Decision makers are typically members of multiple social groups. Consider, for example, an economics professor. She belongs to an economics department and may belong to a group of faculty who regularly get together to play poker. As a member of these groups, she is a conduit through which information flows not only within a group but also from one group to another. She may learn, for example, from fellow poker players about university health plans, and she may transmit this information via her choice of plan to her colleagues in the economics department (and vice versa). Similarly, information flows both within and between the divisions of a firm through its senior managers. Information is also conveyed within and between different groups of friends on social networking sites, like Facebook, via friends, friends of friends, and so on, who are members of multiple groups.

The present paper introduces "interacting cascades," a new class of social networks in which information flows between groups. In an interacting cascade, there are several different groups of decision makers (e.g., economists and poker players), but some decision makers belong to more than one group. It is through these decision makers that information flows between groups, and behavior in one group influences behavior in another.

We study simple interacting cascades in which two groups of players share a common member. Each player chooses whether to adopt or reject a behavior, where it is optimal to adopt if the true (but unknown) state is high and reject if the state is low. Prior to making her decision, each regular player observes an informative private signal as well as the prior decisions of the members of her own group. The common player observes her own private signal and the prior decisions of the members of both groups. Since players moving after the common player observe her decision, such players indirectly learn about the decisions and information of the players in the other group. In this way, information spills over from each group to the other.

For players moving prior to the common player, an interacting cascade is identical in structure to Bikhchandani, Hirshleifer, and Welch (1992)'s model of an information cascade and equilibrium has the same structure: A player follows her own signal, choosing adopt if her signal is high and reject if her signal is low, so long as the difference in the number of adopt and reject decisions by prior players does not exceed one. If, however, the number of adopt decisions exceeds the number of reject decisions by two or more, then a player ignores 
her own signal and also chooses adopt, as the information revealed by the decisions of prior players outweighs the information contained in her own private signal. The player is said to be in a "cascade" on adopt. Likewise, a cascade on reject results when the number of reject decisions exceeds the number of adopt decisions by two or more.

Our focus is on the decisions of the common player and the players who move after her. We show that if an information cascade forms in only one group prior to the move of the common player, then the cascade spills over via the common player to the other group. If, however, the groups are in cascades on different actions, then the common player breaks the cascade in one group. In this case, the common player follows her own signal, the cascade that agrees with the common player's decision continues, and the cascade that disagrees with the common player's decision ends. Finally, if neither group is in a cascade, then the common player follows her own signal, and the common player's decision triggers cascades (on her decision) in both groups.

Perhaps surprisingly, information spillovers via a common player need not enhance welfare. Intuitively, this occurs because the common player suppresses learning when her decision triggers cascades in both groups. We provide, however, sufficient conditions for information spillovers to be welfare enhancing. In particular, the payoff of every player is weakly higher in an interacting cascade if either four or more players in each group move prior to the common player or the players' private signals are sufficiently informative.

This finding indicates that the common player's position has implications for welfare if her turn to move comes too early, then she shuts down learning prematurely, while if it comes too late, then fewer players benefit from her aggregation of information. The optimal position of the common player balances these two effects. We conclude with an analysis of this optimal position. We show that if the signal precision is high (and thus the benefit from extending information aggregation is small), then total surplus is maximized when the common player's turn to move comes early, while if it is low then surplus is maximized when the common player moves late.

Our results shed light on the how the structure of social networks affects the adoption of products and behaviors. Lindstrom and Muñoz-Franco's (2005) study of contraceptive use in Guatemala provides an example of our result that if a cascade emerges in one group but not the other, then interaction results in the cascade spilling. The authors find that urban migrants (the common players) transmit the urban convention of contraceptive use 
back to their (rural) villages, where contraceptive use was previously sporadic. Closer to home, Javorcik's (2004) study of spillovers from foreign direct investment in Lithuania also provides an example of this result. He finds that ventures by foreign firms from developed countries (the common players) are able to transmit productivity-enhancing practices to their up-stream Lithuanian suppliers and those suppliers' competitors.

The balance of this section discusses the related literature. Section 2 introduces our model of interacting cascades. Section 3 provides an illustrative example. Section 4 states our results and Section 5 concludes. Appendix A contains the proof of existence and uniqueness of equilibrium. The main results are established in Appendix B, while computational details are relegated to the online Appendix C.

\section{Related Literature}

Our work is partially an extension of the observational learning literature. As its name suggests, this literature is concerned with how players learn from the behavior of others. A typical model assumes that players move sequentially and face payoffs that depend on the unknown state. When a player moves, she makes a one-time decision after observing some common signal, which is often the complete history of prior decisions, and a private signal. She uses this information to make inferences about the state and pick a payoff-maximizing action.

In their seminal paper, Bikhchandani et al. (1992) found that a cascade eventually forms and, with positive probability, it forms on the action that is incorrect for the realized state. Subsequently, much of the literature has concerned itself with characterizing when players asymptotically learn the true state under different signal structures (if they ever do). For instance, Cao et al. (2011) allow (heterogeneous) players to observe the decisions and payoffs of their predecessors and show that players may never learn the true state, Celen and Kariv (2001) only allow players to observe their immediate predecessor's action and show that beliefs and decisions cycle, and Smith and Sorensen (2000) show that learning obtains when private signals are arbitrarily informative. See Bikhchandani et al. (1998) for an overview of early work. More recent include Acemoglu et al. (2011), Banerjee and Fudenberg (2004), Callander and Horner (2009), Guarino et al. (2011), Guarino and Jehiel (2009), Larson (2008), and Smith and Sorensen (2008). ${ }^{1}$ Our work differs in that our focus is on group

\footnotetext{
${ }^{1}$ Experimental and empirical studies have found support for cascades (in smaller groups) and observational
} 
interaction, not asymptotic learning.

Our work is also related to Bala and Goyal (1998), Gale and Kariv (2003), and MuellerFrank (2013) who examine learning in social networks in which players move repeatedly. In these models, players face payoffs that depend on the unknown state and pick an action to maximize their instantaneous payoff after observing the history of their neighborhoods (and an initial private signal). These papers (i) show that players' decisions, regardless of their positions in the network, asymptotically converge to the optimal action so long as networks are connected, and (ii) they develop conditions under which learning does not obtain. While these models allow for a greater variety of social networks than ours, they don't focus on how the conventions of different groups interact.

In related papers, Acemoglu et al. (2014) and Golub and Jackson (2010) examine games where players first get private signals and then make one-time decisions after sharing their private information over their (endogenously chosen) social networks. Both papers develop sufficient conditions for asymptotic learning: Acemoglu et al. (2014) shows that players must be close to "information hubs," while Golub and Jackson (2010) show that the weight players attach to each person's information must be limited.

More broadly, our work is related to a literature that studies factors that drive the emergence of conventions. For instance, Bernheim (1994) points out that conventions may be explained by a desire for conformity. In his static model, players get utility from social status and consumption. In equilibrium, non-conformity in consumption is punished by a loss of status, so players have an equilibrium desire to conform. Bernheim characterizes when this desire is strong enough to cause a convention to emerge. Other authors have also used a desire for conformity to explain the emergence of conventions - e.g., Akerlof (1997) and Becker (1991).

Jackson and Yariv (2007) point out that best-reply dynamics of network games can also drive the emergence and propagation of conventions. Young (1996) and the papers therein take a related view. They examine evolutionary coordination games where agents have limited memories and they show that conventions may emerge as a way of solving coordination problems. They characterize the nature of these conventions and examine how learning in general. See Anderson and Holt (1997), Drehmann et al. (2005), Cai et al. (2009), and Weizsacker (2010) among others. Goeree et al. (2007) experimentally demonstrate the fragility of cascades in larger groups (e.g., 20+ participants). 
they migrate on networks. Our findings are complementary as we approach the issue of interaction from different economic and technical perspectives. In particular, we focus on the effects of information flows, whereas Young focuses on coordination.

Our interaction results are related to Cipriani and Guarino (2008), who examine asset price cascades with two correlated assets and show that a cascade in one market may spillover into the other market. We differ in that our focus is on social networks rather than financial markets. In particular, our players only observe the history of decisions in their own groups as opposed to the history of prices/decisions in both markets. This leads to differences in our interaction results - e.g., Cipriani and Guarino show that learning stops forever once both markets are in cascades, while we find that if both groups are in different cascades, then interaction results in learning resuming.

\section{The Model}

This section describes the environment, the equilibrium concept, and establishes that an equilibrium exists and is essentially unique.

\section{Basic Cascades}

In a (basic) cascade, $N$ identical players move sequentially in a commonly known and exogenous order. Let $i$ denote the $i$-th player to move, where $i \in\{1,2, \ldots, N\}$. When it is her move, a player decides whether to adopt $(a)$ or reject $(r)$ a behavior. A player's payoff depends only on her own decision and the true, but unknown state, which may be either high $(\mathcal{H})$ and low $(\mathcal{L})$. The payoff of player $i$ when she takes decision $d_{i} \in\{a, r\}$ in state $s \in\{\mathcal{H}, \mathcal{L}\}$ is

$$
u\left(d_{i}, s\right)=\left\{\begin{aligned}
1 & \text { if } d_{i}=a \text { and } s=\mathcal{H} \\
-1 & \text { if } d_{i}=a \text { and } s=\mathcal{L} \\
0 & \text { if } d_{i}=r .
\end{aligned}\right.
$$

Each state is equally likely, i.e., $P(\mathcal{H})=P(\mathcal{L})=1 / 2$. Each player $i$ observes the decisions of the prior players $\bar{d}_{i-1}:=\left(d_{1}, \ldots, d_{i-1}\right)$ and an informative private signal $x_{i} \in\{H, L\}$, prior to making her own decision. The probability of signal $x$, conditional on the true state being $s$, is

$$
P(x \mid s)=\left\{\begin{array}{cc}
p & \text { if } x=s \\
1-p & \text { if } x \neq s
\end{array}\right.
$$


where $p \in(1 / 2,1)$. Given $s$, the signals $x_{i}$ and $x_{j}$ are independent when $i \neq j$. We write $\bar{x}_{i}$ for $\left(x_{1}, \ldots, x_{i}\right)$, where $\bar{x}_{0}=\emptyset$.

A belief for player $i$ is a function $\phi_{i}:\{a, r\}^{i-1} \times\{H, L\} \rightarrow[0,1]$ which gives the probability she assigns to the state being $\mathcal{H}$ given the decisions of the prior players and her own signal. A (pure) strategy for player $i$ is a function $\sigma_{i}:\{a, r\}^{i-1} \times\{H, L\} \rightarrow\{a, r\}$, which maps the profile of decisions of prior players and her own private signal into a decision.

\section{Interacting CASCADES}

We study interacting cascades in which there are two groups - $A$ and $B$ - each with $N$ players. In each group, the players move sequentially in a commonly known and exogenous order. We write $i^{g}$ for the $i$-th player to move in group $g \in\{A, B\}$. The groups interact via a common player who is a member of both groups and who is the $k$-th player to move in each group. For simplicity, we take $k$ to be odd and $1<k<N .^{2}$ The arrangement of the players is illustrated in Figure 1. Each player observes the decisions of the prior players in her own group and an informative private signal prior to making her own decision. The common player observes the decisions of the prior players in both groups.

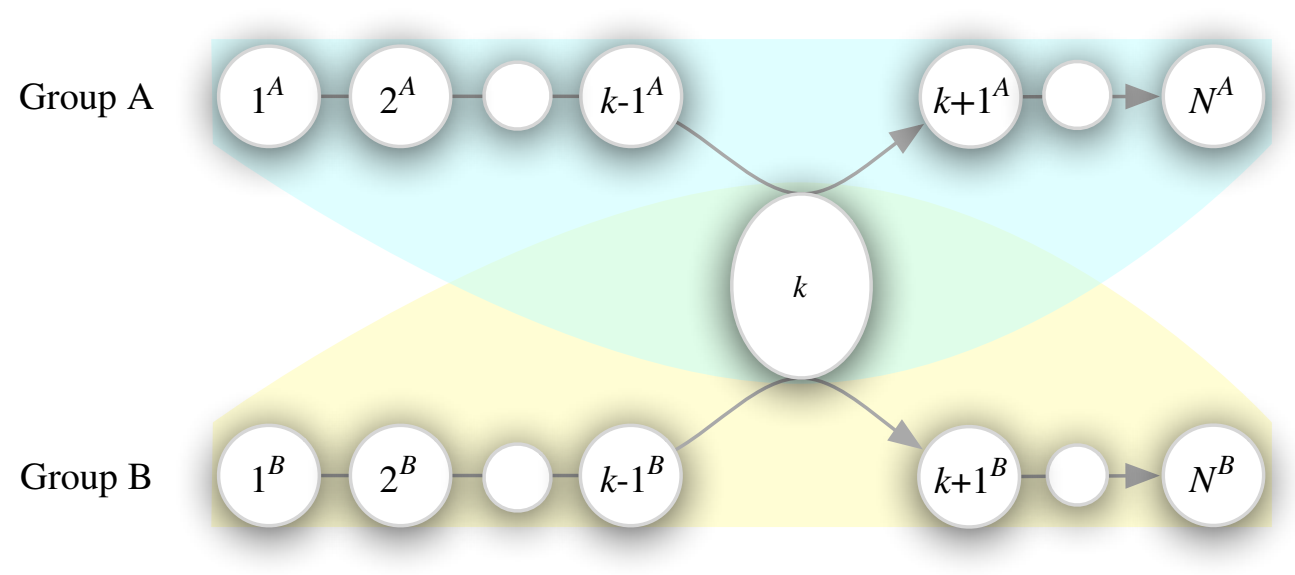

Figure 1: An Interacting Cascade

For each player other than the common player, strategies and beliefs are as defined for a basic cascade. For the common player $k$ a belief is a function $\phi_{k}:\{a, r\}^{2(k-1)} \times\{H, L\} \rightarrow[0,1]$ which gives the probability she assigns to the true state being $\mathcal{H}$ given the decision of the $2(k-1)$ prior players and her private signal. A (pure) strategy for player $k$ is a function

\footnotetext{
${ }^{2}$ We discuss the case where $k$ is even in the Conclusion.
} 
$\sigma_{k}:\{a, r\}^{2(k-1)} \times\{H, L\} \rightarrow\{a, r\}$, which maps the decisions of the prior players and her own private signal into a decision.

We write $x_{i}^{g}$ for the signal of the $i$-th player in group $g$, we write $\phi_{i}^{g}$ for her belief, $d_{i}^{g}$ for her decision, and $\sigma_{i}^{g}$ for her strategy. Since player $k$ is a member of both groups, we denote her decision by either $d_{k}^{A}, d_{k}^{B}$, or simply $d_{k}$. We write $\bar{d}_{i}^{g}$ for $\left(d_{1}^{g}, \ldots, d_{i}^{g}\right)$ for the decisions of players 1 through $i$ in group $g$.

A strategy profile $\sigma$ is a list

$$
\left(\sigma_{1}^{A}, \sigma_{1}^{B}, \ldots, \sigma_{k-1}^{A}, \sigma_{k-1}^{B}, \sigma_{k}, \sigma_{k+1}^{A}, \sigma_{k+1}^{B}, \ldots, \sigma_{N}^{A}, \sigma_{N}^{B}\right)
$$

and a belief profile $\phi$ is a list

$$
\left(\phi_{1}^{A}, \phi_{1}^{B}, \ldots, \phi_{k-1}^{A}, \phi_{k-1}^{B}, \phi_{k}, \phi_{k+1}^{A}, \phi_{k+1}^{B}, \ldots, \phi_{N}^{A}, \phi_{N}^{B}\right)
$$

Given a strategy profile $\sigma$, the probability that the decision profile of the first $i$ players in group $g$ is $\bar{d}_{i}=\left(\bar{d}_{i-1}, d_{i}\right) \in\{a, r\}^{i}$ when the true state is $s$ is denoted by $P_{\sigma}^{g}\left(\bar{d}_{i} \mid s\right)$, and is defined recursively. For $g \in\{A, B\}$ and $i=0$, we define $\bar{d}_{0}=\varnothing$ and $P_{\sigma}^{g}\left(\bar{d}_{0} \mid s\right)=1$. For $g \in\{A, B\}$ and $i=1$ we have

$$
P_{\sigma}^{g}\left(\bar{d}_{1} \mid s\right)=\sum_{\left\{x \mid \sigma_{1}^{g}(x)=d_{1}\right\}} P(x \mid s)
$$

For $g \in\{A, B\}$ and $i \in\{2, \ldots, N\} \backslash\{k\}$ we have

$$
P_{\sigma}^{g}\left(\bar{d}_{i-1}, d_{i} \mid s\right)=P_{\sigma}^{g}\left(\bar{d}_{i-1} \mid s\right) \sum_{\left\{x \mid \sigma_{i}^{g}\left(\bar{d}_{i-1}, x\right)=d_{i}\right\}} P(x \mid s) .
$$

For $g=A$ and $i=k$ we have

$$
P_{\sigma}^{A}\left(\bar{d}_{k-1}, d_{k} \mid s\right)=P_{\sigma}^{A}\left(\bar{d}_{k-1} \mid s\right) \sum_{\bar{d}_{k-1}^{B} \in\{a, r\}^{k-1}} P_{\sigma}^{B}\left(\bar{d}_{k-1}^{B} \mid s\right) \sum_{\left\{x \mid \sigma_{k}\left(\bar{d}_{k-1}, \bar{d}_{k-1}^{B}, x\right)=d_{k}\right\}} P(x \mid s),
$$

whereas for $g=B$ and $i=k$ we have

$$
P_{\sigma}^{B}\left(\bar{d}_{k-1}, d_{k} \mid s\right)=P_{\sigma}^{B}\left(\bar{d}_{k-1} \mid s\right) \sum_{\bar{d}_{k-1}^{A} \in\{a, r\}^{k-1}} P_{\sigma}^{A}\left(\bar{d}_{k-1}^{B} \mid s\right) \sum_{\left\{x \mid \sigma_{k}\left(\bar{d}_{k-1}^{A}, \bar{d}_{k-1}, x\right)=d_{k}\right\}} P(x \mid s) .
$$

Definition: An equilibrium is a profile of (pure) strategies and beliefs $\left(\sigma^{*}, \phi^{*}\right)$ such that $\sigma^{*}$ is sequentially rational and $\phi^{*}$ satisfies Bayes' rule (on the equilibrium path). 
Appendix A provides a formal definition of equilibrium. Sequential rationality requires that each player's decision be optimal given her belief. If $\phi_{i}^{g *}\left(\bar{d}_{i-1}, x\right)>1 / 2$, then player $i$ 's (expected) payoff to choosing $a$ is

$$
u(a, \mathcal{H}) \phi_{i}^{g *}\left(\bar{d}_{i-1}, x\right)+u(a, \mathcal{L})\left(1-\phi_{i}^{g *}\left(\bar{d}_{i-1}, x\right)\right)=\phi_{i}^{g *}\left(\bar{d}_{i-1}, x\right)-\left(1-\phi_{i}^{g *}\left(\bar{d}_{i-1}, x\right)\right)>0
$$

whereas her payoff to choosing $r$ is zero. Hence, $a$ is optimal. If $\phi_{i}^{g *}\left(\bar{d}_{i-1}, x\right)<1 / 2$, then $r$ is optimal. If $\phi_{i}^{g *}\left(\bar{d}_{i-1}, x\right)=1 / 2$, then $a$ and $r$ both yield a payoff of zero and are optimal. In this case, our definition requires (as is common in the literature) that player $i$ follows her own signal, choosing $a$ if she observes $H$ and $r$ if she observes $L$.

Proposition 1. Existence and Uniqueness of Equilibrium.

A (pure) strategy equilibrium exists. Equilibrium is unique up to off-equilibrium-path beliefs, i.e., fix a profile of private signals $\left(x_{1}^{A}, x_{1}^{B}, \ldots, x_{k}, \ldots, x_{N}^{A}, x_{N}^{B}\right)$, then the equilibrium outcome $\left(d_{1}^{A}, d_{1}^{B}, \ldots, d_{k}, \ldots, d_{N}^{A}, d_{N}^{B}\right)$ is the same in every equilibrium.

The players moving prior to the common player face the same game in an interacting cascade and in a basic cascade. For these players, equilibrium play coincides with the equilibrium play for the basic cascade given in Bikhchandani et al. (1992).

We need one definition before discussing the illustrative example in the next section.

Definition: Player $i$ is in a cascade given (pure) strategy $\sigma_{i}$ and history $\bar{d}_{i-1}$ if her action does not depend on her private signal, i.e., if $\sigma_{i}\left(\bar{d}_{i-1}, H\right)=\sigma_{i}\left(\bar{d}_{i-1}, L\right)$.

Observe that if player $i$ in group $g$ is not in a cascade, then subsequent players in group $g$ can infer her signal from her decision.

\section{An Illustrative Example}

To illustrate our model and give intuition for our core results, we consider an example wherein a group of co-workers, A(lice), B(ill), D(avid), E(mma), M(ichael), S(ue), and W(alter), each decide whether to watch Netflix's latest new show on the night it is released. The show may either be good (state $\mathcal{H}$ ) or bad (state $\mathcal{L}$ ) with equal probability. The players would like to watch a good show, i.e., choose $a$ if the state is $\mathcal{H}$, and avoid watching a bad show, i.e., choose $r$ if the state is $\mathcal{L}$. 
The problem is that no one knows the quality of the show before it is released. However, everyone has seen a promo about it (e.g., a billboard, online clip, etc.), i.e., they've each received an informative private signal. For the sake of argument, we assume that the promo correctly indicates the quality of the show with $\frac{2}{3}$-rds probability, i.e., $p=\frac{2}{3}$. In addition, some of the coworkers subscribe to each other's Netflix "Social" feeds, and can observe whether their peers are also watching the show. ${ }^{3}$ Specifically, Bill, David, and Walter, collectively the Men, all subscribe to each other's feeds. Likewise, the Alice, Emma, and Sue, collectively the Women, all subscribe to each other's feeds. Michael subscribes to everyone's feed.

Once Netflix releases the show, the co-workers make their viewing decisions in alphabetical order. When a player moves, (s)he observes the feeds (s)he's subscribed to and her signal, and then makes her decision. To round out the example, we suppose that watching a good show pays 1 , while not watching pays 0 , and watching a bad show pays -1 . The arrangement of players is illustrated in Figure 2.

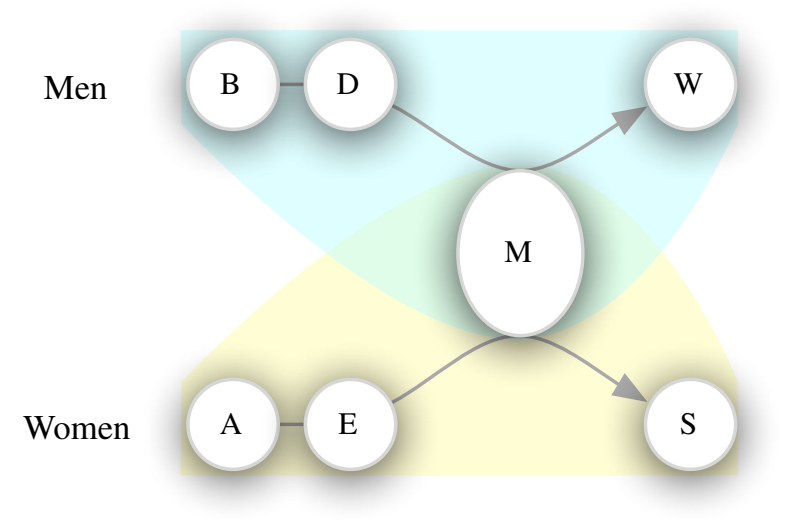

Figure 2: Arrangement of Players in Example

\section{BASIC CASCADES}

To see how a cascade forms, we focus on the Men and suppose, for the moment, that the Women do not exist. Suppose that Bill's (private) signal is $L$, i.e., the promo he watched

\footnotetext{
${ }^{3}$ Netflix offers its subscribers the ability to automatically share what they watch to social media sites (e.g., Facebook), via a Netflix Social feed. See www.netflix.com for details. While this feed also shows one's ratings, we have in mind an environment where players are making their decisions so quickly that they have yet to rate the show.
} 
indicates that Netflix's show is likely bad. Then, his belief is

$$
\phi_{B}^{*}(L)=\frac{P(\mathcal{H}) P(L \mid \mathcal{H})}{\sum_{s \in\{\mathcal{H}, \mathcal{L}\}} P(s) P(L \mid s)}=\frac{\frac{1}{2}(1-p)}{\frac{1}{2}(1-p)+\frac{1}{2} p}=\frac{1}{3},
$$

and he optimally chooses not to watch, i.e., $\sigma_{B}^{*}(L)=r$. If B's signal had been $H$, then $\phi_{B}^{*}(H)=2 / 3$ and B would have chosen $a$. B's decision thus reveals his signal to David, Michael, and Walter. Note that in state $\mathcal{H}$ the probability $\mathrm{B}$ chooses $r$ is $P_{\sigma^{*}}(r \mid \mathcal{H})=1-p=$ $1 / 3$, while in state $\mathcal{L}$ we have $P_{\sigma^{*}}(r \mid \mathcal{L})=p=2 / 3$.

David sees Bill choose $r$ and infers that B received a signal of $L$. If D's signal is also $L$ (i.e., $\bar{d}_{B}=r$ and $x_{D}=L$ in the notation of the model) then his belief is

$$
\phi_{D}^{*}(r, L)=\frac{P(\mathcal{H}) P(L \mid \mathcal{H}) P_{\sigma^{*}}(r \mid \mathcal{H})}{\sum_{s \in\{\mathcal{H}, \mathcal{L}\}} P(s) P(L \mid s) P_{\sigma^{*}}(r \mid s)}=\frac{\frac{1}{2}(1-p)^{2}}{\frac{1}{2}(1-p)^{2}+\frac{1}{2} p^{2}}=\frac{1}{5} .
$$

Thus, D also chooses $r$. If D's signal had been $H$, then $\phi_{D}^{*}(r, H)=1 / 2$ and $\mathrm{D}$ would have chosen $a$, following his signal. D's action therefore also reveals his signal.

Michael, after observing Bill and David choose not to watch, i.e., $\bar{d}_{D}=(r, r)$, infers that they both received signals of $L$. Thus,

$$
\phi_{M}^{*}\left(r, r, x_{M}\right)= \begin{cases}3 / 7 & \text { if } x_{M}=H \\ 1 / 9 & \text { if } x_{M}=L,\end{cases}
$$

which is less than $1 / 2$ for both realizations of $x_{M}$. Hence, $\mathrm{M}$ optimally follows the decisions of $\mathrm{B}$ and $\mathrm{D}$ and also chooses $r$. Consequently, $\bar{d}_{M}=(r, r, r)$. As M's decision does not depend on his signal, his decision is uninformative to Walter. Therefore, W has the same belief as M, i.e., $\phi_{W}^{*}\left(\bar{d}_{M}, x\right)=\phi_{M}^{*}\left(\bar{d}_{D}, x\right)$ for $x \in\{L, H\}$, and also chooses $r$.

When Bill and David both choose $r$, then Michael and Walter are in a cascade. M chooses $r$ because the information revealed by B and D's decisions is strong enough to "outweigh" his signal. Since M's decision is uninformative, then $\mathrm{W}$ is in the same situation as $\mathrm{M}$ and makes the same decision. This leads to our first observation.

Observation 1: In a basic cascade, if the private signals of the first two players (e.g., Bill and David) are the same, then a cascade emerges, i.e., the third player and all subsequent players make the same decision as the first two players.

If the signals of the first two players are not the same, then Michael is not in a cascade. If David's signal had been $H$ rather than $L$, he would have chosen $a$. In this case, M infers 
$\bar{x}_{D}=(L, H)$ and has a belief

$$
\phi_{M}^{*}\left(r, a, x_{M}\right)= \begin{cases}2 / 3 & \text { if } x_{M}=H \\ 1 / 3 & \text { if } x_{M}=L .\end{cases}
$$

Thus, M's decision would depend on his signal.

\section{InteraCting CASCADES}

To see how cascades interact, we return to our initial setting with both Men and Women. In light of Observation 1, we say that group $g$ is in a cascade if the decisions of the first two players in $g$ are the same. We illustrate equilibrium play for the common player and the subsequent players when: (i) one group is in a cascade and the other is not, and (ii) both groups are in cascades on different actions.

To examine (i), suppose that the Men are in a cascade on not watching, i.e., both Bill and David choose $r$, and that the Women are not in a cascade, e.g., Alice chooses $a$ and Emma chooses $r$. Thus, $\bar{d}_{D}^{M}=(r, r)$ and $\bar{d}_{E}^{W}=(a, r)$. Since these players follow their private signals, Michael infers that $\bar{x}_{D}^{M}=(L, L)$ and $\bar{d}_{E}^{W}=(H, L)$. M's belief given his signal $x_{M}$ is

$$
\phi_{M}^{*}\left(\bar{d}_{D}^{M}, \bar{d}_{E}^{W}, x_{M}\right)= \begin{cases}\frac{\frac{1}{2} p^{2}(1-p)^{3}}{\frac{1}{2} p^{2}(1-p)^{3}+\frac{1}{2} p^{3}(1-p)^{3}}=\frac{1}{3} & \text { if } x_{M}=H \\ \frac{\frac{1}{2} p(1-p)^{4}}{\frac{1}{2} p(1-p)^{4}+\frac{1}{2} p^{4}(1-p)}=\frac{1}{9} & \text { if } x_{M}=L .\end{cases}
$$

Thus, M decides $r$, regardless of his signal, following the action of the group that's in a cascade.

Table 1 summarizes how Michael's (equilibrium) decision depends on his signal and the private signals of Bill, David, Alice, and Emma. M will choose $r$ if at least three of these

\begin{tabular}{|c|c|c|c|c|c|c|c|c|}
\hline \multirow{3}{*}{$\operatorname{Men}\left(x_{B}, x_{D}\right)$} & \multicolumn{4}{|c|}{$x_{M}=H$} & \multicolumn{4}{|c|}{$x_{M}=L$} \\
\hline & \multicolumn{4}{|c|}{ Women $\left(x_{A}, x_{E}\right)$} & \multicolumn{4}{|c|}{ Women $\left(x_{A}, x_{E}\right)$} \\
\hline & $H H$ & $H L$ & $L H$ & $L L$ & $H H$ & $H L$ & $L H$ & $L L$ \\
\hline$H H$ & $a$ & $a$ & $a$ & $a$ & $a$ & $a$ & $a$ & $r$ \\
\hline$H L$ & $a$ & $a$ & $a$ & $r$ & $a$ & $r$ & $r$ & $r$ \\
\hline$L H$ & $a$ & $a$ & $a$ & $r$ & $a$ & $r$ & $r$ & $r$ \\
\hline$L L$ & $a$ & $r$ & $r$ & $r$ & $r$ & $r$ & $r$ & $r$ \\
\hline
\end{tabular}
five signals are $L$ and will choose $a$ otherwise.

Table 1: Michael's Decisions 
Continuing the example, suppose that Michael indeed chooses $r$. Walter observes $\bar{d}_{M}^{M}=$ $(r, r, r)$, from which he infers that $x_{B}=x_{D}=L$. He cannot infer $\left(x_{A}, x_{E}, x_{M}\right)$. However, W knows that the only signal profiles, $\left(x_{B}, x_{D}, x_{A}, x_{E}, x_{M}\right)$, which are consistent with $\bar{d}_{M}^{M}$ are those in the last row of Table 1 except for the left-most cell $(L, L, H, H, H)$. W calculates that

$$
P_{\sigma^{*}}^{M}\left(\bar{d}_{M}^{M} \mid \mathcal{H}\right)=3 p^{2}(1-p)^{3}+3 p(1-p)^{4}+(1-p)^{5}=\frac{19}{243}
$$

and

$$
P_{\sigma^{*}}^{M}\left(\bar{d}_{M}^{M} \mid \mathcal{L}\right)=3 p^{3}(1-p)^{2}+3 p^{4}(1-p)+p^{5}=\frac{104}{243} .
$$

W's belief, given signal $x_{W}$, is ${ }^{4}$

$$
\phi_{W}^{*}\left(\bar{d}_{M}^{M}, x_{W}\right)= \begin{cases}\frac{19}{71} & \text { if } x_{W}=H \\ \frac{19}{227} & \text { if } x_{W}=L .\end{cases}
$$

Thus, W chooses $r$, regardless of his signal. This leads to our second observation.

Observation 2a: If group $g$ is in a cascade and the other group is not, then the decision of the common player follows the cascade and the cascade in $g$ continues.

Let's consider the Women. Sue observes $\bar{d}_{M}^{W}=(a, r, r)$ and infers $\bar{x}_{E}^{W}=(H, L)$. Since Michael decided $r, \mathrm{~S}$ knows that there were at least three $L$ signals among $\left(x_{B}, x_{D}, x_{A}, x_{E}, x_{M}\right)$. Since $x_{A}=H$ and $x_{E}=L$, then $\mathrm{S}$ knows that Bill, David, and Michael collectively received at least two $L$ signals. The probability of this is $3 p(1-p)^{2}+(1-p)^{3}$ if the state is $\mathcal{H} .^{5} \mathrm{~S}$ calculates that

$$
P_{\sigma^{*}}^{W}\left(\bar{d}_{M}^{W} \mid \mathcal{H}\right)=p(1-p)\left[3 p(1-p)^{2}+(1-p)^{3}\right]=\frac{14}{243}
$$

and, in an analogous manner, that

$$
P_{\sigma^{*}}^{W}\left(\bar{d}_{M}^{W} \mid \mathcal{L}\right)=p(1-p)\left[3 p^{2}(1-p)+p^{3}\right]=\frac{40}{243} .
$$

S's belief, given signal $x_{S}$, is

$$
\phi_{S}^{*}\left(\bar{d}_{M}^{W}, x_{S}\right)=\left\{\begin{array}{cl}
\frac{7}{17} & \text { if } x_{S}=H \\
\frac{7}{47} & \text { if } x_{S}=L
\end{array}\right.
$$

\footnotetext{
${ }^{4}$ For instance,
}

$$
\phi_{W}^{\star}\left(\bar{d}_{M}^{M}, H\right)=\frac{\frac{1}{2} p P_{\sigma^{*}}^{M}\left(\bar{d}_{M}^{M} \mid \mathcal{H}\right)}{\frac{1}{2} p P_{\sigma^{*}}^{M}\left(\bar{d}_{M}^{M} \mid \mathcal{H}\right)+\frac{1}{2}(1-p) P_{\sigma^{*}}^{M}\left(\bar{d}_{M}^{M} \mid \mathcal{L}\right)}=\frac{19}{71} .
$$

${ }^{5}$ There are three ways that two of the players could receive an $L$ signal, and one way all three players could receive an $L$ signal. 
Thus, S chooses $r$ regardless of her signal. Intuitively, M's action conveys enough information about the state to outweigh S's private signal and the information revealed by Alice and Emma's decisions. The cascade on $r$ "spills over" from the Men to the Women. This leads to our third observation.

Observation 2b: If group $g$ is in a cascade and the other group $-g$ is not, then subsequent players in $-g$ follow the cascade. That is, the cascade spills over from one group to the other.

We now consider the case in which both groups are in cascades on different actions. Suppose that the Men are in a cascade on not watching, i.e., both Bill and David choose $r$, and that the Women are in a cascade on watching, i.e., both Alice and Emma choose a. Then Michael infers $\bar{x}_{D}^{M}=(L, L)$ and $\bar{x}_{E}^{W}=(H, H)$. Table 1 shows that $\mathrm{M}$ resolves his decision in favor of his signal.

Suppose that $x_{M}=L$ and thus $d_{M}=r$. Then $\bar{d}_{M}^{M}=(r, r, r)$ and Walter's belief is given by (1), so he optimally chooses $r$ and the cascade in the Men's group continues. Sue, however, observes $\bar{d}_{M}^{W}=(a, a, r)$ and infers $\bar{x}_{E}^{W}=(H, H)$. Table 1 shows that when $x_{A}=x_{E}=H$, then Michael only chooses $r$ if $\left(x_{B}, x_{D}, x_{M}\right)=(L, L, L)$. Hence, S's history effectively contains three $L$ signals and two $H$ signals. S's belief, given signal $x_{S}$, is

$$
\phi_{S}^{*}\left(\bar{d}_{M}^{W}, x_{S}\right)= \begin{cases}\frac{1}{2} & \text { if } x_{S}=H \\ \frac{1}{5} & \text { if } x_{S}=L .\end{cases}
$$

Hence, $\mathrm{S}$ chooses $a$ if $x_{S}=H$ and $r$ if $x_{S}=L$, and so $\mathrm{S}$ is not in a cascade. Intuitively, M's decision to not watch conveys enough information to $\mathrm{S}$ for her to doubt her group's cascade on watching, but not enough to give rise to a new cascade. This leads to our next observation.

Observation 3: If both groups are in cascades on different actions, then the cascade that agrees with the common player's action continues, while the cascade that disagrees with the common player's action ends.

Observations 2a, 2b, and 3 allow us to write Table 2, a description of how cascades interact when the Men are in a cascade on $a$. In the table, " $a$ " denotes a cascade on $a$, " $r$ " a cascade on $r$, and " $n / a$ " denotes no cascade. For instance, the second row of the 
table shows that if the Men are in a cascade on a (before Michael moves) and the Women aren't in a cascade, then $\mathrm{M}$ chooses $a$, the Men remain in a cascade on $a$, and this cascade is transmitted to the Women.

\begin{tabular}{|c|c|c|c|c|}
\hline \multicolumn{2}{|c|}{ Existing Cascade } & \multirow[b]{2}{*}{$d_{M}$} & \multicolumn{2}{|c|}{ Subsequent Cascac } \\
\hline Men & Women & & Men & Women \\
\hline \multirow{4}{*}{$a$} & $a$ & $a$ & $a$ & $a$ \\
\hline & $n / a$ & $a$ & $a$ & $a$ \\
\hline & $r$ & $a$ & $a$ & $n / a$ \\
\hline & $r$ & $r$ & $n / a$ & $r$ \\
\hline
\end{tabular}

Table 2: Michael's Effect on Cascades in Both Groups

Table 2 shows our two findings for this example: (i) if one group is in a cascade and the other is not, then the cascade is transmitted through the common player to the second group, and (ii) when two cascades on opposite actions meet, one ends. It also shows that when both groups cascade on the same action, then interaction has no effect.

\section{WELFARE}

When is interaction via a common player welfare improving? Table 2 indicates that the common player Michael sometimes generates a benefit. For instance, if the state is $\mathcal{H}$, the Men are in cascade on $a$ (the correct action), and the Women are in a cascade on $r$ (the incorrect action), then when M chooses $a$ he ends the Women's incorrect cascade and raises Sue's payoff. This comes with the risk, however, that M may choose $r$ and end the Men's correct cascade, thereby decreasing Walter's payoff. To get a handle on this we need to compare the (ex-ante) equilibrium payoffs of Walter and Sue with and without a common player.

In a basic cascade, where Michael is a regular player, Walter's (and Sue's) equilibrium payoff is $w^{B}=13 / 54$. In an interacting cascade, where $\mathrm{M}$ is a common player, W's equilibrium payoff is $w^{I}=47 / 162$. Since $w^{I}-w^{B}=4 / 81>0, \mathrm{~W}$ and $\mathrm{S}$ are better off when groups interact than when they do not. ${ }^{6}$

The presence of a common player raises payoffs by increasing the chance that players who follow him choose the correct action. He accomplishes this by (i) ending incorrect cascades

\footnotetext{
${ }^{6}$ Equilibrium payoffs in basic and interacting cascades are given in Proposition 5.
} 
more often than correct cascades and (ii) by transmitting correct cascades (to a group not in a cascade) more often than incorrect cascades. For this illustrative example, we can show that interaction always raises welfare. We will establish the following result in general.

Observation 4: Ex-ante (equilibrium) payoffs in the interacting cascade are (strictly) higher than ex-ante payoffs in the basic cascade if $p$ is sufficiently high or $k$ is sufficiently large.

\section{Results}

In this section, we characterize equilibrium in interacting cascades. We also evaluate the welfare consequences of the information spillovers resulting from the presence of a common player, and we consider the welfare-maximizing placement of the common player.

Proposition 2 gives the behavior of players prior to the common player. Since, prior to the common player, an interacting cascade is identical to the basic cascade introduced in Bikhchandani et al. (1992), Proposition 2 follows from their results.

Proposition 2. In equilibrium, a player moving before the common player chooses a regardless of her own signal (i.e., is in a cascade on a) if the number of a decisions by her predecessors exceeds the number of $r$ decisions by two or more. Similarly, a player chooses $r$ regardless of her own signal if the number of $r$ decisions by her predecessors exceeds the number of a decisions by two or more. Otherwise, a player follows her own signal, i.e., she chooses a given signal $H$ and $r$ given signal $L$.

According to the proposition, if the number of a decisions by a player's predecessors exceeds the number of $r$ decisions by two or more, then the player herself also chooses $a$. All the players successors (among those who move before the common player) therefore also choose $a$, regardless of their private signal. Hence, there is no further information aggregation.

We say that a group is in a cascade on $\boldsymbol{a}(\boldsymbol{r})$ if the number of $a(r)$ decisions by the first $k-1$ players in the group exceeds the number of $r(a)$ decision by two or more. Proposition 3 identifies the equilibrium behavior of the common player.

Proposition 3. In equilibrium, the common player chooses a (likewise $r$ ) regardless of her own signal if either (i) both groups are in a cascade on a $(r)$, or (ii) one group is in a 
cascade on a $(r)$ and the other group is not in a cascade. Otherwise, the common player follows her own signal, i.e., she chooses a given the signal $H$ and $r$ given the signal $L$.

According to the proposition, the common player's action depends on her signal if neither group is in a cascade or both groups are in cascades on different actions. Otherwise, the common player herself ignores her own signal. A player in position $i>k$ knows only whether her own group was in a cascade prior to the common player, and thus can not tell whether the common player is in a cascade.

Proposition 4 is our principle result. It identifies the conditions under which (i) a cascade "spills over" from one group to another, (ii) information spillovers end an existing cascade, and (iii) the common player's decision "triggers" a new cascade, i.e., begins a cascade where there was none before.

Proposition 4: In equilibrium, if prior to the common player:

P4.1 : Both groups are in cascades on a, then each cascade continues, i.e., the common player and all subsequent players in both groups choose a.

P4.2 : One group is in a cascade on a and the other group is not in a cascade, then the cascade on a "spills over" to the other group, i.e., the common player and all the subsequent players in both groups choose $a$.

P4.3 : Both groups are in cascades on different actions, then (i) the common player follows her own signal, (ii) the cascade that agrees with the common player's action continues, and (iii) the cascade that disagrees with the common player's action ends, i.e., player $k+1$ in the group whose cascade disagrees with the common player's action follows her own signal. P4.4 : Neither group is in a cascade, then the common player "triggers" two cascades, i.e., the common player follows her own signal and all subsequent players in both groups choose the same action as the common player.

The analogous statements apply to cascades on $r$.

Proposition 5 gives each player's equilibrium payoff in an interacting cascade. 
Proposition 5. In an interacting cascade, the equilibrium payoff of player $i$ is

$$
w(i, k)= \begin{cases}\frac{2 p-1}{2} \Lambda(i+1) & \text { for } i<k \text { and odd } \\ \frac{2 p-1}{2} \Lambda(2 k)+(2 p-1)\left[\frac{\lambda}{2} \Lambda(k-1)\right]^{2} & \text { for } i=k \\ \frac{2 p-1}{2} \Lambda(2 k)+(2 p-1)\left[\frac{\lambda}{2} \Lambda(k-1)\right]^{2}\left[1+\frac{\lambda}{2} \Lambda(i-k)\right] & \text { for } i>k \text { and odd, }\end{cases}
$$

where $\lambda=2 p(1-p)$ and

$$
\Lambda(i)=\frac{1-\lambda^{\frac{i}{2}}}{1-\lambda} .
$$

Furthermore, $w(i+1, k)=w(i, k)$ for $i$ odd, i.e., the even player moving immediately after an odd player obtains the same payoff as the odd player.

We write $w^{B}(i)$ for the payoff of the $i$-th player to move in a basic cascade. One can show that $w^{B}(i)=\frac{2 p-1}{2} \Lambda(i+1)$ and $w^{B}(i+1)=w^{B}(i)$ for odd $i$. Players moving prior to the common player get the same payoff in an interacting cascade and a basic cascade since, for them, an interacting cascade is a basic cascade.

The equilibrium payoffs of players $i$ and $i+1$ are the same when $i$ is odd. For $i \neq k$ this is a consequence of the fact that $i$ and $i+1$ are either (i) both in the same cascade or (ii) both follow their own signal. For $i=k$, this is also a consequence of (iii) that when neither group is in a cascade the common player's decision triggers a cascade, causing player $k+1$ to make the same decision as player $k$.

Let $c(i, k)$ denote the probability that player $i$ correctly guesses the true state in equilibrium, i.e., chooses $a$ if the state is $H$ and chooses $r$ if the state is $L$. Player $i$ 's payoff $w(i, k)$ can be written as $\frac{1}{2} c(i, k)+\frac{1}{2}[-(1-c(i, k))]=c(i, k)-1 / 2$, i.e., ${ }^{7}$

$$
c(i, k)=w(i, k)+1 / 2 .
$$

Since $\Lambda(i)$ is increasing, the following is an immediate corollary of Proposition 5.

Corollary 1. Players positioned later in an interacting cascade obtain higher payoffs and have a higher probability of choosing the optimal action given the true state. More precisely, the payoff of every odd player is strictly greater than the payoff of the odd player who immediately precedes her.

\footnotetext{
${ }^{7}$ If the true state is $H$, with probability $c(i, k)$ player $i$ 's chooses $a$ and obtains 1 and chooses $r$ and with probability $1-c(i, k)$ obtains 0 . If the true state is $L$, player $i$ obtains 0 with probability $c(i, k)$ and -1 with probability $1-c(i, k)$.
} 


\section{The Welfare Consequences of Information Spillovers}

Our next results compare player payoffs in interacting and basic cascades, and thus shed light on the costs and benefits of information spillovers between groups. From Proposition 5 it is clear that $w(k, k)>w^{B}(k)$, i.e., the common player in an interacting cascade obtains a higher payoff than her counterpart in a basic cascade. The intuition for this result is straightforward: The common player has better information. Since she observes both groups, she infers at least two more signals than her counterpart does in a basic cascade.

One might conjecture that the introduction of a common player raises the payoff of every player who moves subsequently relative to what that player would obtain in a basic cascade. This conjecture is not correct as the following example illustrates. Figure 3 below shows player payoffs as a function of position in an interacting cascade (with $k=3$ and $p=.6$ ) and in a basic cascade.

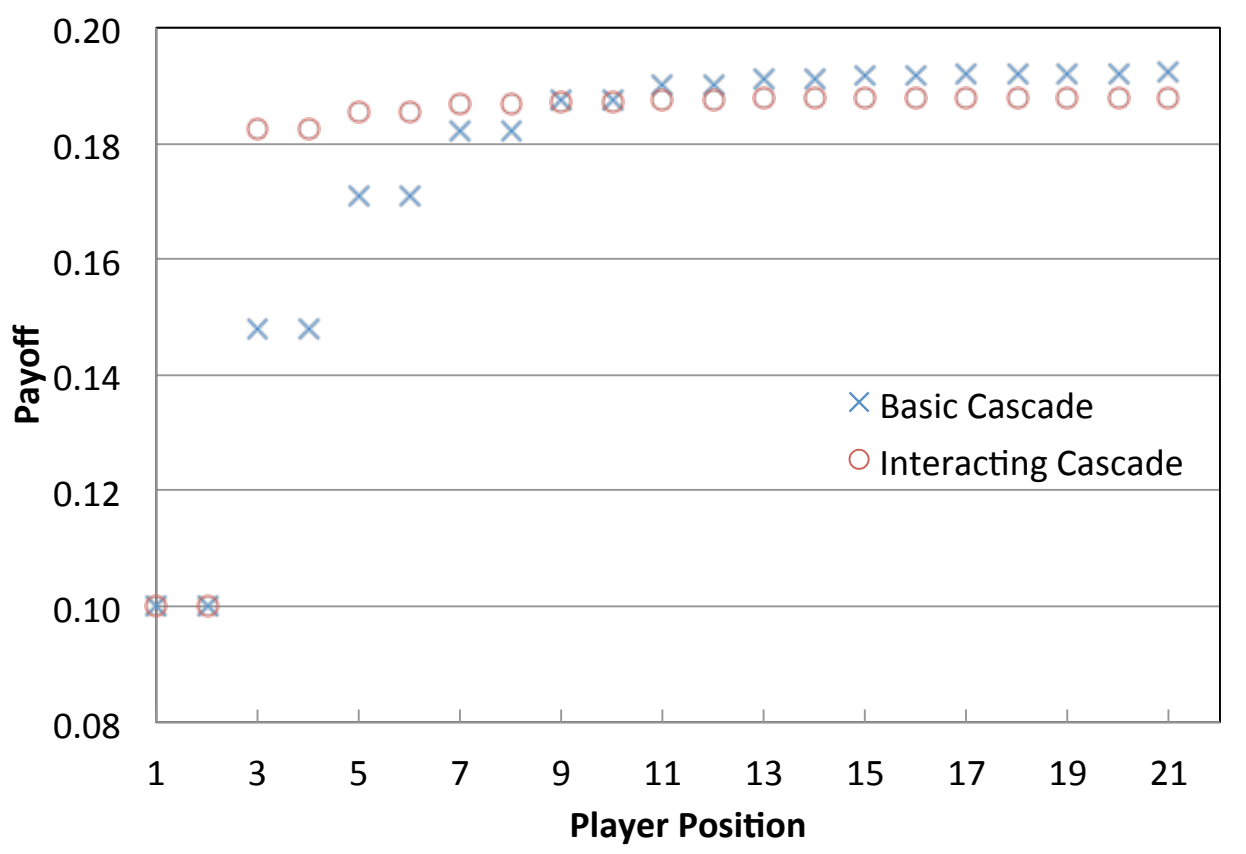

Figure 3: Payoffs by Position, $k=3$ and $p=0.4$

In this example, players who move late in the basic cascade have higher payoffs than players in the same position in the interacting cascade. For Player 11, for instance, $w^{B}(11) \approx$ $0.189956>w(11,5) \approx 0.187595$, and every player who moves subsequently has a higher payoff in the basic cascade.

Player 11 is affected in multiple ways (some good and others bad) by the information 
spillovers that occur through the common player. First, these spillovers may cause an existing cascade to end. If players $1^{A}$ and $2^{A}$ both make the correct decision, then in the basic cascade game Player $3^{A}$ and every subsequent player is in a cascade and makes the correct decision as well. In contrast, in the interacting cascade game, if Players $1^{A}$ and $2^{A}$ both make the correct decision, then Player $11^{A}$ makes a correct decision only with probability .9622. In particular, if group $B$ is in a cascade on the incorrect action, the information spillover may end the correct cascade in group A, thereby lowering Player $11^{A}$ 's payoff. Conversely, information spillovers may end an incorrect cascade. If players $1^{A}$ and $2^{A}$ both make the incorrect decision, then in the basic cascade game every subsequent player makes the incorrect decision as well. In contrast, in an interacting cascade Player $11^{A}$ makes the correct decision with probability .1885 .

A more subtle effect of information spillovers is their potential to suppress positive information externalities. Suppose that Players $1^{A}$ and $2^{A}$ make opposing decisions. In the basic cascade game, Player $3^{A}$ follows her own signal and chooses the correct action with probability $p$ (.6 in this example). Player $11^{A}$, however, makes the correct decision with a higher probability of .6874. The difference between these probabilities is the positive information externality that Player $11^{A}$ enjoys from observing the decisions of players $3^{A}$ through $10^{A}$. In the interacting cascade, by contrast, when players $1^{A}$ and $2^{A}$ make opposing decision, the players in group $A$ are certain to be in an information cascade on the common player's decision, and thus enjoy no additional information externalities. ${ }^{8}$ The probability that Player $11^{A}$ makes the correct decision is only .6480.

The strength of these effects depends on the signal accuracy and the location of the common player. If $k=5$, then the probability that Player $11^{A}$ makes the correct decision, conditional on players $1^{A}$ to $4^{A}$ following their own signals, is almost the same in the basic cascade (.6821) and an interacting cascade (.6710). Likewise, the probability that a cascade on the correct action is upset rises to .0566 , while the probability that a cascade on the incorrect action is upset rises to .2776 .

Proposition 6 identifies conditions under which the common player aggregates information in a strong sense - she obtains a higher payoff than every player in a basic cascade. In particular, so long as either information sharing does not occur "too early" or private signals

\footnotetext{
${ }^{8}$ In particular, either a cascade in $B$ spills over to group $A$ (see $\mathrm{P} 4.2$ ) or the common player triggers a cascade in $A$ (see $\mathrm{P} 4.4)$.
} 
are sufficiently informative, then a player is better off by being the common player (and observing the decision of $k-1$ member of each group) than she is by being in any position in a basic cascade (and observing the decisions of any number of the prior players in her group). In other words, the information revealed from observing the first $k-1$ decisions in each group is more valuable than the information revealed by observing any number of prior decisions in a basic cascade.

Proposition 6. The payoff of the common player in an interacting cascade exceeds the payoff of every player in a basic cascade, i.e., $w(k, k)>w^{B}(i)$ for every $i \in\{1, \ldots, \infty\}$, if either $k \geq 5$ or $k=3$ and $p \geq \frac{1}{6} \sqrt{3}+\frac{1}{2}$. If $k=3$ and $p<\frac{1}{6} \sqrt{3}+\frac{1}{2}$, then there is an $i^{\prime}$ such that $w(k, k)<w^{B}(i)$ for $i \geq i^{\prime}$.

Since payoffs are higher for players moving later in the cascade, i.e., since $w(i, k)$ is increasing in $i$, we have for $i>k$ that

$$
w(i, k) \geq w(k, k)>w^{B}(i)
$$

The next corollary is immediate.

Corollary 2. If either $k \geq 5$ or $k=3$ and $p \geq \frac{1}{6} \sqrt{3}+\frac{1}{2}$ then the payoff of every player $i \geq k$ is strictly higher in an interacting cascade than in a basic cascade.

Under the assumptions of Corollary 2, the presence of a common player is welfare enhancing in a strong sense: the common player and every one of her successors in an interacting cascade is better off than a player in the same position in a basic cascade. Since the payoff of players moving before the common player is the same as in a basic cascade, we conclude that everyone is (weakly) better off with information spillovers than without information spillovers. $^{9}$

\section{Placement of the Common Player}

\footnotetext{
${ }^{9}$ We imagine getting rid of information spillovers by removing the common player from group $B$. To be more precise, we name the players by their positions in the interacting cascade. After we remove the common player from group $B$, our groups are $A=\left\{1^{A}, \ldots, k-1^{A}, k, k+1^{A}, \ldots, N^{A}\right\}$ and $B=\left\{1^{B}, \ldots, k-\right.$ $\left.1^{B}, k+1^{B}, \ldots, N^{B}\right\}$. Since the groups are unconnected, they each play a basic cascade where players move according to their initial indices.
} 
If information is shared between groups, when should it be shared? There is a trade-off between the number of players who benefit from information sharing and the quality of the information shared. If the common player moves early, then more players subsequently enjoy the benefits of information spillovers. However, if the common player moves later, she and her successors are better-informed and more likely to take the correct action.

Here we consider positioning the common player in order to maximize total surplus. Total surplus in an interacting cascade is

$$
W(N, p, k)=w(k, k)+\sum_{i \in\{1, \ldots, N\} \backslash\{k\}} 2 w(i, k),
$$

since there is a single common player, two identical players in the $i$-th position of each group, and equilibrium is symmetric. Our objective is to choose $k$, where $1<k<N$ and $k$ is odd, to maximize $W(N, k)$. Let $k^{*}(N, p)$ denote the (smallest) solution. If $k=1$, the total surplus is just twice the surplus of a basic cascade, and thus never maximizes total surplus.

A useful way to proceed is to think about the effect on payoffs of moving the common player from position $k(>3)$ to position $k-2$. We do this by picking up the common player, shifting the players occupying positions $k-2$ and $k-1$ one position to the right to fill in the gap, and then inserting the common player into the now empty $k-2$ position. The top panel of Figure 4 depicts the original game and the bottom depicts the new game. The figure shows, for instance, that player $k-2^{A}$ moves from position $k-2$ in group $A$ to position $k-1$ in group $A$.

We consider the effect on total surplus of this move. The payoffs of players 1 through $k-3$ are the same in both games since they don't change position and their payoffs don't depend on the common player's position (see Proposition 5). However, the payoffs of each of the remaining players change since they depend on the common player's position.

The payoffs of players $k-2$ and $k-1$ increase as they have better information about the state. To see this for player $k-2$, recall that in the original game her payoff is $w(k-2, k)$, while in the new game her payoff is $w(k-1, k-2)$. By Proposition 5 , we have

$$
\begin{aligned}
w(k-2, k) & =\frac{2 p-1}{2} \Lambda(k-1) \\
& <\frac{2 p-1}{2} \Lambda(2 k-4)+(2 p-1)\left[\frac{\lambda}{2} \Lambda(k-3)\right]^{2}\left[1+\frac{\lambda}{2} \Lambda(1)\right] \\
& =w(k-1, k-2),
\end{aligned}
$$




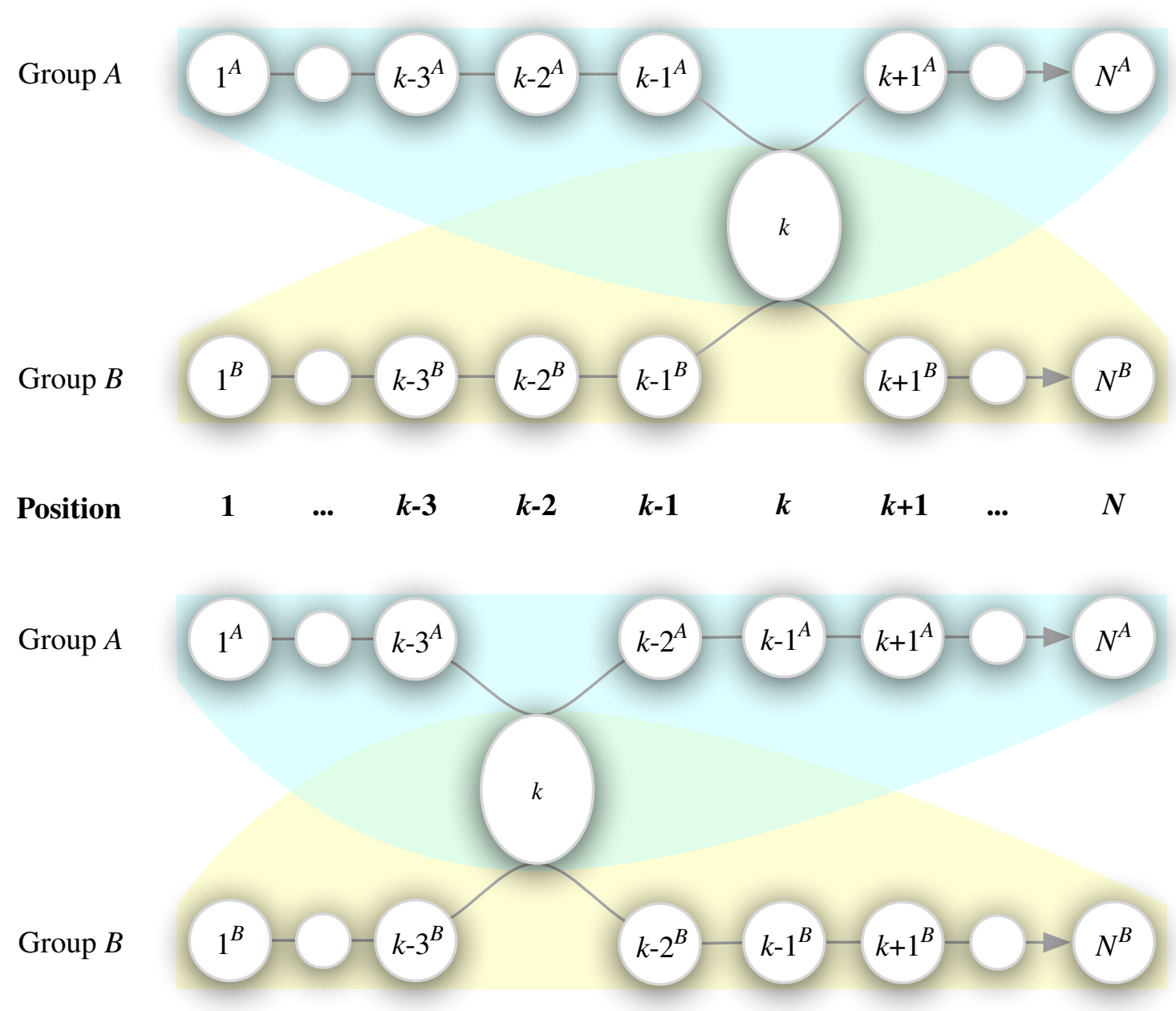

Figure 4: Moving the Common Player

where the strict inequality holds since $\Lambda$ is increasing and $k>3$ implies $2 k-4>k-1$. The common player's payoff decreases as she observes fewer decisions and, thus, less information. The effect on the payoffs of the remaining players is ambiguous since, for $i>k$, the sign of $w(i, k)-w(i, k-2)$ depends on $i$. For instance, if $p=0.6$, then Player 25's surplus increases by 0.00178 (from 0.24952 to 0.25130 ) when the common player is moved from position 21 to position 19, while Player 29's surplus decreases by 0.00003 (from 0.25300 to 0.25297). One can show $W(29, .6,21)=11.1836$ and $W(29, .6,19)=11.4281$, and hence the effect on total surplus is positive when $N=29$. The effect on total surplus, however, can be made negative if $N$ is made sufficiently large.

Proposition 7 shows that, for $p$ sufficiently close to one, total surplus increases when the common player moves from position $k$ to $k-2$ (as in Figure 4 ). In other words, when the 
signal accuracy is high, then the benefit of realizing information spillovers earlier exceeds the cost of reduced information aggregation.

Proposition 7. For each odd $k>3$, there exists a $p_{k}<1$ such that moving the common player from position $k$ to $k-2$ increases total surplus when $p \in\left[p_{k}, 1\right)$, i.e., $W(N, p, k-2)>$ $W(N, p, k)$ for all $p \in\left[p_{k}, 1\right)$.

It follows immediately that $k^{*}(N, p)=3$ for $p$ sufficiently close to 1 . In other words, when the signal accuracy is high, then the gains to aggregating the information of more than the first two players in each group are more than offset by the benefits from realizing information spillovers immediately.

Corollary 3. There is a $p_{N}<1$ such that $k^{*}(N, p)=3$ for all $p \in\left[p_{N}, 1\right)$.

Table 3 illustrates how $W(N, p, k)$ depends on $p$ and $k$ when $N=15$ (i.e., there are 14 players in each group and one common player).

\begin{tabular}{lllllll}
$p$ & $k=3$ & $k=5$ & $k=7$ & $k=9$ & $k=11$ & $k=13$ \\
\hline 0.55 & 2.583 & 2.900 & $\mathbf{2 . 9 2 8}$ & 2.851 & 2.737 & 2.617 \\
\hline 0.65 & 7.342 & 8.033 & $\mathbf{8 . 0 5 4}$ & 7.865 & 7.611 & 7.351 \\
\hline 0.75 & 11.016 & $\mathbf{1 1 . 5 6 6}$ & 11.511 & 11.321 & 11.102 & 10.883 \\
\hline 0.85 & 13.275 & $\mathbf{1 3 . 4 4 0}$ & 13.371 & 13.278 & 13.183 & 13.089 \\
\hline 0.95 & $\mathbf{1 4 . 2 7 3}$ & 14.266 & 14.255 & 14.245 & 14.234 & 14.223 \\
\hline
\end{tabular}

Bold indicates a maximum

Table 3: $W(15, p, k)$

If $p=0.55$, for example, then total surplus is maximized with the common player in position 7. The table illustrates that, as signals become more informative, the optimal position of the common player moves earlier.

There is a weak, "dual" result to Corollary 3: for each integer $n \geq 3$, there is a $p^{\prime}>\frac{1}{2}$ such that $k^{*}(N, p)>n$ for all $p \in\left(\frac{1}{2}, p^{\prime}\right]$ and $N$ sufficiently large. That is, it is best to wait before aggregating information when $p$ is sufficiently close to $\frac{1}{2}$ and a large number of players move after the third position. This result is not particularly surprising in light of our previous discussion and Table 3, so we omit the proof. 


\section{Conclusion}

We have shown that when groups share members in common then: (i) a convention may spillover from one group to another via the common player; (ii) a convention in a group will be broken if it disagrees with the action of the common player; and (iii) a convention can be triggered by the action of common player when no prior convention existed.

We conclude with a discussion of the robustness of our results. Our assumption that the each player's signal has the same precision is not essential. If instead the signal precisions of the members of groups $A$ and $B$ (excluding the common player) are $p^{A}$ and $p^{B}$, respectively, and that of the common player is $p$, then Propositions 1 through 4 go through without modification provided that $p^{A}, p^{B}$, and $p$ are close. Since the equilibrium is invariant to small changes in the signal precisions, the equilibrium payoffs (Proposition 5) are continuous functions of $p^{A}, p^{B}$, and $p$ in the neighborhood of $p^{A}=p^{B}=p$. Thus, as in Proposition

6 , either $k \geq 5$ or $p>\frac{1}{6} \sqrt{3}+\frac{1}{2}$ are sufficient for the common player's payoff to exceed the payoff of every player in a basic cascade, provided $p^{A}$ and $p^{B}$ are in a neighborhood of $p$. Propositions 1 through 4 also go through without modification if the numbers of players in each group that move prior to the common player are unequal (so long as the common player has an odd position in each group). However, the expression for payoffs in Proposition 5 relies on the symmetry of the baseline model, and thus one would need to develop new expressions for payoffs and new sufficient conditions for the payoff of the common player to exceed those of all the other players.

The structure of equilibrium is more complex when the common player is in an even position. The reason is that an odd number of players move before the common player in each group. Thus, when the common player observes that neither group is in a cascade, her posterior belief is no longer uniform, but rather depends on the decisions of players $k-1^{A}$ and $k-1^{B}$. That said, our results remain qualitatively the same — in particular, interaction still results in the spillover, breakage, and triggering of cascades under very similar circumstances. Results are available upon request. 


\section{References}

[1] Acemoglu, D., Bimpkis, K., and A. Ozdaglar, Dynamics of Information Exchange in Endogenous Social Networks, Theoretical Economics (2014) 9, 41-97.

[2] Acemoglu, D., Dahleh, M., Lobel, I., and A. Ozdaglar, Bayesian Learning in Social Networks, The Review of Economic Studies (2011) 78, 1201-1236.

[3] Akerlof, G., Social Distance and Social Decisions, Econometrica (1997), 65, 1005-1027.

[4] Anderson, L. and C. Holt, Information Cascades in the Laboratory, American Economic Review (1997) 87, 847-862.

[5] Bala, V., and S. Goyal, Learning from Neighbours, The Review of Economic Studies (1998) 65 (3), 595-621.

[6] Banerjee, A. and D. Fudenberg, Word-of-Mouth Learning, Games and Economic Behavior (2004) 46, 1-22.

[7] Becker, G., A Note on Restaurant Pricing and Other Examples of Social Influences on Price, Journal of Political Economy (1991) 99, 1109-1116.

[8] Bernheim, B., A Theory of Conformity, Journal of Political Economy (1994), 102, 841-877.

[9] Bikhchandani, S., Hirshleifer, D., and I. Welch, A Theory of Fads, Fashion, Custom, and Cultural Change as Informational Cascades, The Journal of Political Economy (1992), 100, 992-1026.

[10] Bikhchandani, S., Hirshleifer, D. and I. Welch, Learning From the Behavior of Others: Conformity, Fads, and Informational Cascades, The Journal of Economic Perspectives (1998), 12, 151-170.

[11] Cao, H., Han, B., and D. Hirshleifer, Taking the Roal Less Traveled By: Does Conversation Eradicate Pernicious Cascades?, Journal of Economic Theory (2011) 146 (4), 1418-1436. 
[12] Cai, H., Chen, Y., and H. Fang, Observational Learning: Evidence From a Randomized Natural Field Experiment, The American Economic Review (2009) 99, 864-882.

[13] Callander, S., and J. Horner, The Wisdom of the Minority, Journal of Economic Theory (2009) 144 (e2), 1421-1439.

[14] Celen, B. and S. Kariv, Observational Learning Under Imperfect Information, Games and Economic Behavior (2001) 47, 72-86.

[15] Cipriani, M., and A. Guarino, Heard Behavior and Contagion In Financial Markets, The B.E. Journal of Theoretical Economics (2008) 8 (1), article 24.

[16] Drehmann, M., Oechssler, J., and A. Roider, Herding and Contrarian Behavior in Financial Markets: An Internet Experiment, American Economic Review (2005) 95 (5), 1403-1426.

[17] Gale, D. and S. Kariv, Bayesian Learning in Social Networks, Games and Economic Behavior (2003) 45, 329-346.

[18] Goeree, J., Palfrey, T., Rogers, B., and R. McKelvey, Self-Correcting Information Cascades, Review of Economic Studies (2007) 74, 733-762.

[19] Golub, B., and M. Jackson, Naive Learning in Social Networks and the Wisdom of Crowds, American Economic Journal Microeconomics (2010) 2 (1), 1112-1149.

[20] Guarino, A., Harmgart, H., and S. Huck, Aggregate Information Cascades, Games and Economic Behavior (2011) 73, 167-185.

[21] Guarino, A., and P. Jehiel, Social Learning with Coarse Inference, University College of London Working Paper, 2009.

[22] Jackson, M., and L. Yariv, Diffusion of Behavior and Equilibrium Properties in Network Games, American Economic Review Papers and Proceedings (2007) 97 (2), 92-98.

[23] Javorcik, B., Does Foreign Direct Investment Increase the Productivity of Domestic Firms? In Search of Spillovers Through Backward Linkages, The American Economic Review (2004) 94, 605-627. 
[24] Larson, N., In With the New or Out With the Old? Bottlenecks in Social Learning, University of Virginia Working Paper, 2008.

[25] Lindstrom, D., and E. Muñoz-Franco, Migration and the Diffusion of Modern Contraceptive Knowledge and Use in Rural Guatemala, Studies in Family Planning (2005) 4, $277-288$.

[26] Mueller-Frank, M., A General Framework for Rational Learning in Social Networks, Theoretical Economics (2013) 8, 1-40.

[27] Smith, L. and P. Sorensen, Pathological Outcomes of Observational Learning, Econometrica (2000) 68, 371-398.

[28] Weizsacker, G., Do We Follow Others when We Should? A Simple Test of Rational Expectation, American Economic Review (2010) 100, 2340-2360.

[29] Young, H., The Economics of Convention, The Journal of Economic Perspectives (1996) 10, 105-122.

\section{Appendix A: Existence and Uniqueness}

We first provide a formal definition of equilibrium, then we prove Proposition 1.

Definition. An equilibrium is a profile of (pure) strategies and beliefs $\left(\sigma^{*}, \phi^{*}\right)$ such that $\sigma^{*}$ is sequentially rational and $\phi^{*}$ satisfies Bayes' rule:

(i) Sequential Rationality

For $g \in\{A, B\}$, and $i \in\{1, \ldots, N\} \backslash\{k\}$, and $\bar{d}_{i-1} \in\{a, r\}^{i-1}$, and $x \in\{H, L\}$ we have:

$$
\sigma_{i}^{g *}\left(\bar{d}_{i-1}, x\right)= \begin{cases}a & \text { if } \phi_{i}^{g *}\left(\bar{d}_{i-1}, x\right)>\frac{1}{2} \\ r & \text { if } \phi_{i}^{g *}\left(\bar{d}_{i-1}, x\right)<\frac{1}{2}\end{cases}
$$

and

$$
\sigma_{i}^{g *}\left(\bar{d}_{i-1}, x\right)= \begin{cases}a & \text { if } \phi_{i}^{g *}\left(\bar{d}_{i-1}, x\right)=\frac{1}{2} \text { and } x=H \\ r & \text { if } \phi_{i}^{g *}\left(\bar{d}_{i-1}, x\right)=\frac{1}{2} \text { and } x=L\end{cases}
$$


Further, for $\left(\bar{d}_{k-1}^{A}, \bar{d}_{k-1}^{B}\right) \in\{a, r\}^{2(k-1)}$ and $x \in\{H, L\}$ we have:

$$
\sigma_{k}^{*}\left(\bar{d}_{k-1}^{A}, \bar{d}_{k-1}^{B}, x\right)= \begin{cases}a & \text { if } \phi_{k}^{g *}\left(\bar{d}_{k-1}^{A}, \bar{d}_{k-1}^{A}, x\right)>\frac{1}{2} \\ r & \text { if } \phi_{k}^{g *}\left(\bar{d}_{k-1}^{A}, \bar{d}_{k-1}^{B}, x\right)<\frac{1}{2},\end{cases}
$$

and

$$
\sigma_{k}^{*}\left(\bar{d}_{i-1}, x\right)= \begin{cases}a & \text { if } \phi_{k}^{g *}\left(\bar{d}_{k-1}^{A}, \bar{d}_{k-1}^{B}, x\right)=\frac{1}{2} \text { and } x=H \\ r & \text { if } \phi_{k}^{g *}\left(\bar{d}_{k-1}^{A}, \bar{d}_{k-1}^{B}, x\right)=\frac{1}{2} \text { and } x=L .\end{cases}
$$

\section{(ii) Bayes' Rule}

For $g \in\{A, B\}$, and $i \in\{2, \ldots, N\} \backslash\{k\}$, and $\bar{d}_{i-1} \in\{a, r\}^{i-1}$, and $x \in\{H, L\}$ we have that

$$
\phi_{i}^{g *}\left(\bar{d}_{i-1}, x\right)=\frac{P_{\sigma^{*}}^{g}\left(\bar{d}_{i-1} \mid \mathcal{H}\right) P(x \mid \mathcal{H}) P(\mathcal{H})}{\sum_{s \in\{\mathcal{H}, \mathcal{L}\}} P_{\sigma^{*}}^{g}\left(\bar{d}_{i-1} \mid s\right) P(x \mid s) P(s)},
$$

if $P_{\sigma^{*}}^{g}\left(\bar{d}_{i-1} \mid s\right)>0$ for some $s$. Further, for $\left(\bar{d}_{k-1}^{A}, \bar{d}_{k-1}^{B}\right) \in\{a, r\}^{2(k-1)}$ and $x \in\{H, L\}$ we have that

$$
\phi_{k}^{*}\left(\bar{d}_{k-1}^{A}, \bar{d}_{k-1}^{B}, x\right)=\frac{P_{\sigma^{*}}^{A}\left(\bar{d}_{k-1}^{A} \mid \mathcal{H}\right) P_{\sigma^{*}}^{B}\left(\bar{d}_{k-1}^{B} \mid \mathcal{H}\right) P(x \mid \mathcal{H}) P(\mathcal{H})}{\sum_{s \in\{\mathcal{H}, \mathcal{L}\}} P_{\sigma^{*}}^{A}\left(\bar{d}_{k-1}^{A} \mid s\right) P_{\sigma^{*}}^{B}\left(\bar{d}_{k-1}^{B} \mid s\right) P(x \mid s) P(s)},
$$

if $P_{\sigma^{*}}^{A}\left(\bar{d}_{k-1}^{A} \mid s\right) P_{\sigma^{*}}^{B}\left(\bar{d}_{k-1}^{B} \mid s\right)>0$ for some $s$.

Proof of Proposition 1: A strategy profile $\sigma$ implies a mapping $e=\left(e_{1}^{A}, e_{1}^{B}, \ldots, e_{k}\right.$, $\left.\ldots, e_{N}^{A}, e_{N}^{B}\right)$ from signal profiles $x=\left(x_{1}^{A}, x_{1}^{B}, \ldots, x_{k}, \ldots, x_{N}^{A}, x_{N}^{B}\right) \in\{H, L\}^{2 N-1}$ to decision profiles $d=\left(d_{1}^{A}, d_{1}^{B}, \ldots, d_{k}, \ldots, d_{N}^{A}, d_{N}^{B}\right) \in\{a, r\}^{2 N-1}$ as follows: For $i=1, e_{1}^{g}(x)=$ $\sigma_{1}^{g}\left(x_{1}^{g}\right)$. For players $i<k, e_{i}^{g}(x)=\sigma_{i}^{g}\left(e_{1}^{g}(x), \ldots, e_{i-1}^{g}(x), x_{i}^{g}\right)$. For player $k$ as $e_{k}(x)=$ $\sigma_{k}\left(e_{1}^{A}(x), \ldots, e_{k-1}^{A}(x), e_{1}^{B}(x), \ldots e_{k-1}^{B}(x), x_{k}\right)$. For player $k+1$ as $e_{k+1}(x)=\sigma_{k+1}^{g}\left(e_{1}^{g}(x), \ldots\right.$, $\left.e_{k-1}^{g}(x), e_{k}(x), x_{k+1}^{g}\right)$. For players $i>k+1$ as $e_{i}^{g}(x)=\sigma_{i}^{g}\left(e_{1}^{g}(x), \ldots, e_{k-1}^{g}(x), e_{k}(x), e_{k+1}^{g}(x)\right.$, $\left.\ldots, e_{i-1}^{g}(x), x_{i}^{g}\right)$.

We prove that this mapping is the same for every equilibrium, and then we construct a pure strategy equilibrium. The proof is by induction. Without loss of generality, we focus on group $A$. Consider player $1^{A}$. If $x_{1}^{A}=H$ then in any equilibrium since player 1 's beliefs satisfies Bayes' rule, and thus

$$
\phi_{1}^{A *}\left(\bar{d}_{0}, H\right)=\frac{P\left(\bar{d}_{0} \mid \mathcal{H}\right) P(H \mid \mathcal{H}) P(\mathcal{H})}{\sum_{s \in\{\mathcal{H}, \mathcal{L}\}} P\left(\bar{d}_{0_{A}} \mid s\right) P(H \mid s) P(s)}=\frac{\frac{1}{2} p}{\frac{1}{2} p+\frac{1}{2}(1-p)}=p,
$$


as $P\left(\bar{d}_{0} \mid s\right)=1$ for each $s$. Since $p>\frac{1}{2}$, sequential rationality implies $\sigma_{1}^{A *}(H)=a$ in any equilibrium. Analogously, $\phi_{1}^{A *}\left(\bar{d}_{0}, L\right)=1-p$ and thus $\sigma_{1}^{A *}(L)=r$. Thus $e_{1}^{A}(x)=a$ whenever $x_{1}^{A}=H$ and $e_{1}^{A}(x)=r$ whenever $x_{1}^{A}=L$ in every equilibrium. Similarly for $B$.

Assume that the mapping $e$ for the first $i-1$ players $\left(e_{1}^{A}, e_{1}^{B}, \ldots, e_{i-1}^{A}, e_{i-1}^{B}\right)$ is the same for every equilibrium. For each $\bar{d}_{i-1}^{A}$ in the range of $\left(e_{1}^{A}, \ldots, e_{k}, \ldots, e_{i-1}^{A}\right)(x)$, we have that (i) $P_{\sigma}^{A}\left(\bar{d}_{i-1}^{A} \mid s\right)$ is the same for every equilibrium $(\sigma, \phi)$ (as the map $\left(e_{1}^{A}, \ldots, e_{k}, \ldots, e_{i-1}^{A}\right)$ is the same for every equilibrium) and (ii) $P_{\sigma}\left(\bar{d}_{i-1}^{A} \mid s\right)>0$ for each $s$ (since each profile of signals occurs with strictly positive probability in each state). Since $\phi_{i}^{A *}\left(\bar{d}_{i-1}^{A}, x\right)$ satisfies Bayes' Rule in any equilibrium, then

$$
\phi_{i}^{A *}\left(\bar{d}_{i-1}^{A}, x_{i}^{A}\right)=\frac{P_{\sigma}^{A}\left(\bar{d}_{i-1}^{A} \mid \mathcal{H}\right) P\left(x_{i}^{A} \mid \mathcal{H}\right) P(\mathcal{H})}{\sum_{s \in\{\mathcal{H}, \mathcal{L}\}} P_{\sigma}^{A}\left(\bar{d}_{i-1}^{A} \mid s\right) P\left(x_{i}^{A} \mid s\right) P(s)}
$$

for each $x_{i}^{A} \in\{H, L\}$ and is the same in every equilibrium. Furthermore, sequentially rational implies that for each $x_{i}^{A} \in\{H, L\}$ we have that

$$
\sigma_{i}^{A *}\left(\bar{d}_{i-1}^{A}, x_{i}^{A}\right)= \begin{cases}a & \text { if } \phi_{i}^{A *}\left(\bar{d}_{i-1}^{A}, x_{i}^{A}\right)>\frac{1}{2}, \text { or } x_{i}^{A}=H \text { and } \phi_{i}^{A *}\left(\bar{d}_{i-1}^{A}, x_{i}^{A}\right)=\frac{1}{2} \\ r & \text { if } \phi_{i}^{A *}\left(\bar{d}_{i-1}^{A}, x_{i}^{A}\right)<\frac{1}{2}, \text { or } x_{i}^{A}=L \text { and } \phi_{i}^{A *}\left(\bar{d}_{i-1}^{A}, x_{i}^{A}\right)=\frac{1}{2},\end{cases}
$$

and is the same in every equilibrium. Thus $e_{i}^{A}(x)=\left(\bar{d}_{i-1}, \sigma_{i}^{A *}\left(\bar{d}_{i-1}, x_{i}^{A}\right)\right)$, where $\bar{d}_{i-1}^{A}=$ $\left(e_{1}^{A}, \ldots, e_{i-1}^{A}\right)(x)$, is the same in every equilibrium. Similarly for $B$. Hence, the equilibrium mapping from signal profiles to decision profiles is uniquely determined: fixing a profile of private signals $x$, then the equilibrium outcome $d$ is the same in every equilibrium. (When $i$ is the common player, an analogous argument applies, save that we must consider histories $\bar{d}_{k-1}^{A}$ and $\bar{d}_{k-1}^{B}$ in the ranges of $\left(e_{1}^{A}, \ldots, e_{k-1}^{A}\right)$ and $\left.\left(e_{1}^{B}, \ldots, e_{k-1}^{B}\right).\right)$

It remains to be shown that an equilibrium exists. Consider group $A$. For each $i$, $x_{i}^{A} \in\{H, L\}$, and $\bar{d}_{i-1}^{A}$ such that $P_{\sigma}\left(\bar{d}_{i-1}^{A} \mid s\right)>0$, let $\phi$ and $\sigma$ be given as above. For $\bar{d}_{i-1}^{A}$ such that $P_{\sigma}\left(\bar{d}_{i-1}^{A} \mid s\right)=0$, let $\phi_{i}^{A}\left(\bar{d}_{i-1}^{A}, x_{i}^{A}\right)=1 / 2$ and let $\sigma_{i}^{A}\left(\bar{d}_{i-1}^{A}, x_{i}^{A}\right)$ be sequentially rational given $\phi_{i}^{A}\left(\bar{d}_{i-1}^{A}, x_{i}^{A}\right)$, i.e., player $i$ follows her own signal. The construction for group $B$ and the common player is analogous. Then $(\sigma, \phi)$ satisfies sequential rationality and Bayes rule by construction. Thus an equilibrium exists. 


\section{Appendix B: Characterization of Equilibrium}

We begin with a few definitions, present four lemmas which completely characterize the players' equilibrium play, and the prove all of our results, except for Proposition 1 . The proofs of these key lemmas are tedious and are given in the online Appendix C.

A history $\bar{d}_{i}=\left(d_{1}, \ldots, d_{i}\right)$ is balanced if, for every odd integer $j<i$, we have that $d_{j} \neq d_{j+1}$, and is unbalanced otherwise. A balanced history is one where the number of $a$ decisions does not exceed the number of $r$ decisions by more than one as of any player $1, \ldots, i$. Any singleton or null history is trivially balanced. For instance, $(a)$ and $(r, a, a, r)$ are balanced, whereas $(a, r, a, a)$ and $(r, r, r)$ are unbalanced. Let $D_{i}^{b}$ be the set of all balanced $i$-length histories.

Let $i>1$. A history $\bar{d}_{i}=\left(d_{1}, \ldots, d_{i}\right)$ is unbalanced on $\boldsymbol{a}$ if at some point in the profile it switches from a balanced profile to a profile of all $a$ s, i.e., if there is an odd $j<i$ such that (i) $\left(d_{1}, \ldots, d_{j}\right)$ is balanced and (ii) $d_{j}=d_{j+1}=\cdots=d_{i}=a$. For instance, $(a, a)$ and $(a, r, a, a)$ are unbalanced on $a$, whereas $(a, r, a, a, r)$ is not unbalanced on $a$. Let $D_{i}^{a}$ be the set of all $i$-length histories that are unbalanced on $a$. Analogously, we say that a history $\bar{d}_{i}=\left(d_{1}, \ldots, d_{i}\right)$ is unbalanced on $\boldsymbol{r}$ if there is an odd $j<i$ such that $(\mathrm{i})\left(d_{1}, \ldots, d_{j}\right)$ is balanced and (ii) $d_{j}=d_{j+1}=\cdots=d_{i}=r$. Let $D_{i}^{r}$ be the set of all $i$-length histories that are unbalanced on $r$. (As we'll make clear, player $j+1$ is the last player to follow her signal in equilibrium.)

Lemma B1. Equilibrium play for predecessors of the common player.

Let $i<k$ and $g \in\{A, B\}$. If $\bar{d}_{i-1}^{g}$ belongs to a row in Table B1(a), then player $i^{g}$ 's equilibrium strategy $\sigma_{i}^{g *}\left(\bar{d}_{i-1}^{g}, x_{i}^{g}\right)$ is given by the last two columns. Otherwise, $\sigma_{i}^{g *}\left(\bar{d}_{i-1}^{g}, H\right)$ 
and $\sigma_{i}^{g *}\left(\bar{d}_{i-1}^{g}, L\right)$ are arbitrary.

\begin{tabular}{lcc} 
& \multicolumn{2}{c}{$\sigma_{i}^{g *}\left(\bar{d}_{i-1}^{g}, x_{i}^{g}\right)$} \\
\cline { 2 - 3 } $\bar{d}_{i-1}^{g}$ & $H$ & $L$ \\
\hline$D_{i-1}^{a}$ & $a$ & $a$ \\
\hline$D_{i-1}^{b}$ & $\mathbf{a}$ & $\mathbf{r}$ \\
\hline$D_{i-1}^{r}$ & $r$ & $r$ \\
\hline
\end{tabular}

(a) Player $i^{g}<k$

\begin{tabular}{cccc} 
& & \multicolumn{2}{c}{$\sigma_{k}^{*}\left(\bar{d}_{k-1}^{A}, \bar{d}_{k-1}^{B}, x_{k}\right)$} \\
\cline { 2 - 4 } $\bar{d}_{k-1}^{A}$ & $\bar{d}_{k-1}^{B}$ & $H$ & $L$ \\
\hline$D_{k-1}^{a}$ & $D_{k-1}^{a}$ & $a$ & $a$ \\
\cline { 2 - 4 } & $D_{k-1}^{b}$ & $a$ & $a$ \\
\cline { 2 - 4 } & $D_{k-1}^{r}$ & $\mathbf{a}$ & $\mathbf{r}$ \\
\hline \multirow{2}{*}{$D_{k-1}^{b}$} & $D_{k-1}^{a}$ & $a$ & $a$ \\
\cline { 2 - 4 } & $D_{k-1}^{b}$ & $\mathbf{a}$ & $\mathbf{r}$ \\
\cline { 2 - 4 } & $D_{k-1}^{r}$ & $r$ & $r$ \\
\hline$D_{k-1}^{r}$ & $D_{k-1}^{a}$ & $\mathbf{a}$ & $\mathbf{r}$ \\
\cline { 2 - 4 } & $D_{k-1}^{b}$ & $r$ & $r$ \\
\cline { 2 - 4 } & $D_{k-1}^{r}$ & $r$ & $r$ \\
\hline
\end{tabular}

(b) Player $k$

Table entries in bold indicate when a player follows her signal.

Table B1: Equilibrium Strategies of Players 1 through $k$.

Proof. Given in Appendix C.

For instance, if player $i$ observes a history that is unbalanced on $a$ then she chooses $a$, ignoring her own signal (i.e., if $\bar{d}_{i}^{g} \in D_{i-1}^{a}$, then Table B1(a) shows that $\sigma_{i}^{g *}\left(\bar{d}_{i-1}^{g}, H\right)=$ $\left.\sigma_{i}^{g *}\left(\bar{d}_{i-1}^{g}, L\right)=a\right)$. If player $i$ observe a balanced history, then she follows her own signal.

Lemma B2. Equilibrium play for the Common Player.

If $\left(\bar{d}_{k-1}^{A}, \bar{d}_{k-1}^{B}\right)$ belongs to a row in Table B1(b), then the common player's equilibrium strategy $\sigma_{k}^{*}\left(\bar{d}_{k-1}^{A}, \bar{d}_{k-1}^{B}, x_{k}\right)$ is given by the last two columns. Otherwise, $\sigma_{k}^{*}\left(\bar{d}_{k-1}^{A}, \bar{d}_{k-1}^{B}, H\right)$ and $\sigma_{k}^{*}\left(\bar{d}_{k-1}^{A}, \bar{d}_{k-1}^{B}, L\right)$ are arbitrary.

Proof. Given in Appendix C.

The next proposition identifies the behavior of players moving after the common player.

Lemma B3. Equilibrium After the Common Player.

Let $g \in\{A, B\}$. 
B3.1 : (Player $k+1^{g}$.) If $\bar{d}_{k}^{g}=\left(\bar{d}_{k-1}^{g}, d_{k}\right)$ belongs to a row in Table B2(a), then player $k+1^{g}$ 's equilibrium strategy $\sigma_{k+1}^{g *}\left(\bar{d}_{k}^{g}, x_{k+1}^{g}\right)$ is given by the last two columns. Otherwise, $\sigma_{k+1}^{g *}\left(\bar{d}_{k}^{g}, H\right)$ and $\sigma_{k+1}^{g *}\left(\bar{d}_{k}^{g}, L\right)$ are arbitrary.

B3.2 : (Player $k+2^{g}$.) If $\bar{d}_{k+1}^{g}=\left(\bar{d}_{k-1}^{g}, d_{k}, d_{k+1}^{g}\right)$ belongs to a row in Table B2(b), then player $k+2^{g}$ 's equilibrium strategy $\sigma_{k+2}^{g *}\left(\bar{d}_{k+1}^{g}, x_{k+2}^{g}\right)$ is given by the last two columns. Otherwise, $\sigma_{k+2}^{g *}\left(\bar{d}_{k+1}^{g}, H\right)$ and $\sigma_{k+2}^{g *}\left(\bar{d}_{k+1}^{g}, L\right)$ are arbitrary.

B3.3 : (Subsequent players.) Let $i>k+2$. If $\bar{d}_{i-1}^{g}=\left(\bar{d}_{k-1}^{g}, d_{k}, d_{k+1}^{g}, d_{k+2}^{g}, \ldots, d_{i-1}^{g}\right)$ belongs to a row in Table B2(c), then player $i^{g}$ 's equilibrium strategy $\sigma_{i}^{g *}\left(\bar{d}_{i-1}^{g}, x_{i}^{g}\right)$ is given by the last two columns. Otherwise, $\sigma_{i}^{g *}\left(\bar{d}_{i-1}^{g}, H\right)$ and $\sigma_{i}^{g *}\left(\bar{d}_{i-1}^{g}, L\right)$ are arbitrary.

\begin{tabular}{|c|c|c|c|c|c|c|c|c|}
\hline \multirow[b]{3}{*}{$\bar{d}_{k-1}^{g}$} & \multirow[b]{3}{*}{$d_{k}$} & & & \multirow[b]{2}{*}{$\bar{d}_{k-1}^{g}$} & \multirow[b]{2}{*}{$d_{k}$} & \multirow[b]{2}{*}{$d_{k+1}^{g}$} & \multicolumn{2}{|c|}{$\sigma_{k+2}^{g *}\left(\bar{d}_{k+1}^{g}, x_{k+2}^{g}\right)$} \\
\hline & & \multicolumn{2}{|c|}{$\sigma_{k+1}^{g *}\left(\bar{d}_{k}^{g}, x_{k+1}^{g}\right)$} & & & & $H$ & $L$ \\
\hline & & $H$ & $L$ & \multirow{3}{*}{$D_{k-1}^{a}$} & $a$ & $a$ & $a$ & $a$ \\
\hline \multirow[t]{2}{*}{$D_{k-1}^{a}$} & $a$ & $a$ & $a$ & & $r$ & $a$ & $\mathbf{a}$ & $\mathbf{r}$ \\
\hline & $r$ & $\mathbf{a}$ & $\mathbf{r}$ & & $r$ & $r$ & $r$ & $r$ \\
\hline \multirow[t]{2}{*}{$D_{k-1}^{b}$} & $a$ & $a$ & $a$ & \multirow[t]{2}{*}{$D_{k-1}^{b}$} & $a$ & $a$ & $a$ & $a$ \\
\hline & $r$ & $r$ & $r$ & & $r$ & $r$ & $r$ & $r$ \\
\hline \multirow[t]{2}{*}{$D_{k-1}^{r}$} & $a$ & $\mathbf{a}$ & $\mathbf{r}$ & \multirow{3}{*}{$D_{k-1}^{r}$} & $a$ & $a$ & $a$ & $a$ \\
\hline & $r$ & $r$ & $r$ & & $a$ & $r$ & $\mathbf{a}$ & $\mathbf{r}$ \\
\hline \multicolumn{4}{|c|}{ (a) Player $k+1^{g}$} & & $r$ & $r$ & $r$ & $r$ \\
\hline
\end{tabular}

(b) Player $k+2^{g}$ 


\begin{tabular}{|c|c|c|c|c|c|}
\hline \multirow[b]{2}{*}{$\bar{d}_{k-1}^{g}$} & \multirow[b]{2}{*}{$d_{k}$} & \multirow[b]{2}{*}{$d_{k+1}^{g}$} & \multirow[b]{2}{*}{$\left(d_{k+2}^{g}, \ldots, d_{i-1}^{g}\right)$} & \multicolumn{2}{|c|}{$\sigma_{i}^{g *}\left(\bar{d}_{i-1}^{g}, x_{i}^{g}\right)$} \\
\hline & & & & $H$ & $L$ \\
\hline \multirow{5}{*}{$D_{k-1}^{a}$} & $a$ & $a$ & $(a, \ldots, a)$ & $a$ & $a$ \\
\hline & \multirow{3}{*}{$r$} & \multirow{3}{*}{$a$} & $D_{i-k-2}^{a}$ & $a$ & $a$ \\
\hline & & & $D_{i-k-2}^{b}$ & $\mathbf{a}$ & $\mathbf{r}$ \\
\hline & & & $D_{i-k-2}^{r}$ & $r$ & $r$ \\
\hline & $r$ & $r$ & $(r, \ldots, r)$ & $r$ & $r$ \\
\hline \multirow[t]{2}{*}{$D_{k-1}^{b}$} & $a$ & $a$ & $(a, \ldots, a)$ & $a$ & $a$ \\
\hline & $r$ & $r$ & $(r, \ldots, r)$ & $r$ & $r$ \\
\hline \multirow{5}{*}{$D_{k-1}^{r}$} & $a$ & $a$ & $(a, \ldots, a)$ & $a$ & $a$ \\
\hline & \multirow{3}{*}{$a$} & \multirow{3}{*}{$r$} & $D_{i-k-2}^{a}$ & $a$ & $a$ \\
\hline & & & $D_{i-k-2}^{b}$ & $\mathbf{a}$ & $\mathbf{r}$ \\
\hline & & & $D_{i-k-2}^{r}$ & $r$ & $r$ \\
\hline & $r$ & $r$ & $(r, \ldots, r)$ & $r$ & $r$ \\
\hline
\end{tabular}

(c) Player $i^{g}>k+2^{g}$

Table B2: Equilibrium Strategies of Players $k+1$ through $N$.

For instance, if $\bar{d}_{k-1}^{g} \in D_{k-1}^{a}$ and $d_{k}=a$, then the top row of Table B2(a) shows that player $k+1^{g}$ is in a cascade on $a$. The intuition underlying of $B 3.1$ is the same as for Sue and Walter's behavior in the Example. While B3.2 and B3.3 are more complicated, similar intuition underlies them.

Proof. Given in Appendix C.

The following technical lemma is useful. For a set $T \subset D_{i}^{a} \cup D_{i}^{b} \cup D_{i}^{r}$, we define $\operatorname{Prob}(T \mid s)=\sum_{\bar{d}_{i} \in T} P_{\sigma^{*}}\left(\bar{d}_{i} \mid s\right)$, where $\sigma^{*}$ is the strategy profile of an equilibrium.

Lemma B4. Let $i<k$ and $g \in\{A, B\}$. If $\bar{d}_{i}^{g}$ is a history that results from equilibrium play, then $\bar{d}_{i}^{g} \in D_{i}^{a} \cup D_{i}^{b} \cup D_{i}^{r}$. In addition, we have

$$
\begin{aligned}
& \operatorname{Prob}\left(D_{i}^{b} \mid s\right)= \begin{cases}\lambda^{\frac{i}{2}} & \text { for } i \text { even } \\
\lambda^{\frac{i-1}{2}} & \text { for } i \text { odd },\end{cases} \\
& \operatorname{Prob}\left(D_{i}^{a} \mid s\right)= \begin{cases}P(H \mid s)^{2} \Lambda(i) & \text { for } i \text { even } \\
P(H \mid s)^{2} \Lambda(i-1) & \text { for } i \text { odd },\end{cases}
\end{aligned}
$$




$$
\operatorname{Prob}\left(D_{i}^{r} \mid s\right)= \begin{cases}P(L \mid s)^{2} \Lambda(i) & \text { for } i \text { even } \\ P(L \mid s)^{2} \Lambda(i-1) & \text { for } i \text { odd }\end{cases}
$$

Proof. Given in Appendix C.

Proof of Proposition 2. In equilibrium, player $i$ observes a history $\bar{d}_{i-1}$ in either $D_{i-1}^{a}$, $D_{i-1}^{b}$, or $D_{i-1}^{r}$ by Lemma B4. For histories where the number of $a$ decisions exceeds the number of $r$ decisions by two or more, we have $\bar{d}_{i-1} \in D_{i-1}^{a}$ and player $i$ chooses $a$ by Table B1 of Lemma B1. Likewise, for histories $\bar{d}_{i-1} \in D_{i-1}^{r}$ the number of $r$ decisions exceeds the number of $a$ decisions by two or more, and she chooses $r$. Otherwise, player $i$ observes a history in $D_{i-1}^{b}$ in which case she follows her own signal.

Proof of Proposition 3. This result follows directly from Lemmas B2 and B4. By Lemma $\mathrm{B} 4$, in equilibrium, the common player observes a pair of histories $\left(\bar{d}_{k-1}^{A}, \bar{d}_{k-1}^{B}\right) \in\left(D_{k-1}^{a} \cup\right.$ $\left.D_{k-1}^{b} \cup D_{k-1}^{r}\right)^{2}$. It follows, from Lemma B2, that if:

(i) Both groups are in a cascade on $a(r)$, i.e., $\bar{d}_{k-1}^{A}$ and $\bar{d}_{k-1}^{B}$ are in $D_{k-1}^{a}\left(D_{k-1}^{r}\right)$, then the common player chooses $a(r)$.

(ii) One group is in a cascade on $a(r)$ and the other is not in a cascade, i.e., either $\bar{d}_{k-1}^{A} \in D_{k-1}^{a}$ $\left(\bar{d}_{k-1}^{A} \in D_{k-1}^{r}\right)$ and $\bar{d}_{k-1}^{B} \in D_{k-1}^{b}$ or $\bar{d}_{k-1}^{A} \in D_{k-1}^{b}$ and $\bar{d}_{k-1}^{B} \in D_{k-1}^{a}\left(\bar{d}_{k-1}^{B} \in D_{k-1}^{r}\right)$, then the common player chooses $a(r)$.

(iii) Neither group is in a cascade, i.e., $\bar{d}_{k-1}^{A} \in D_{k-1}^{b}$ and $\bar{d}_{k-1}^{B} \in D_{k-1}^{a}$, then the common player follows her signal.

Proof of Proposition 4. We establish each part via iterative application of Lemmas B2, $\mathrm{B} 3$, and B4.

P4.1. Since both groups are in cascades on $a$, we have that $\bar{d}_{k-1}^{A}$ and $\bar{d}_{k-1}^{B}$ are in $D_{k-1}^{a}$ by Lemma B4. Thus, Lemma B2 gives that the common player chooses $a$. Hence successive application of Lemma B3 gives that every subsequent player in group $g$ chooses $a$. To illustrate, consider player $k+1^{g}$ who observes $\left(\bar{d}_{k-1}^{g}, a\right)$ and so chooses a regardless of the value of her signal by Lemma B3.1. It follows that player $k+2^{g}$ observes $\left(\bar{d}_{k-1}^{g}, a, a\right)$ and so chooses $a$ by Lemma B3.2. Player $k+3^{g}$ then observes $\left(\bar{d}_{k-1}^{g}, a, a, a\right)$ and chooses $a$ by Lemma B3.3. Player $k+4^{g}$ thus observes $\left(\bar{d}_{k-1}^{g}, a, a, a, a\right)$ and also chooses $a$ by Lemma 
B3.3. Continuing the application of Lemma B3.3 gives that every subsequent player $k+5^{g}$ to $N^{g}$ also chooses $a$. It follows that the cascade continues in group $g$. Since $g$ was arbitrary, Proposition 4.1 follows.

P4.2. Since one group is in a cascade and the other isn't, by Lemma B4 we have $\bar{d}_{k-1}^{A} \in D_{k-1}^{a}$ $\left(\bar{d}_{k-1}^{A} \in D_{k-1}^{r}\right)$ and $\bar{d}_{k-1}^{B} \in D_{k-1}^{b}$ or $\bar{d}_{k-1}^{A} \in D_{k-1}^{b}$ and $\bar{d}_{k-1}^{B} \in D_{k-1}^{a}\left(\bar{d}_{k-1}^{b} \in D_{k-1}^{r}\right)$. Without loss, we consider the case of $\bar{d}_{k-1}^{A} \in D_{k-1}^{a}$ and $\bar{d}_{k-1}^{B} \in D_{k-1}^{b}$. Lemma B2 gives that the common player chooses $a$. Thus, successive application of Lemma B3 gives that every subsequent player in both groups also chooses $a$.

P4.3. Since both groups are in opposing cascades, by Lemma B4 we have (i) $\bar{d}_{k-1}^{A} \in D_{k-1}^{a}$ and $\bar{d}_{k-1}^{B} \in D_{k-1}^{r}$ or (ii) $\bar{d}_{k-1}^{A} \in D_{k-1}^{r}$ and $\bar{d}_{k-1}^{B} \in D_{k-1}^{a}$. Without loss, we consider case (i). Lemma B2 gives that the common player follows her signal $x_{k}$. Suppose she gets signal $x_{k}=H$ and so chooses $a$ by Lemma B2. Thus, successive application of Lemma B3 gives that every subsequent player in group $A$ chooses $a$. However, player $k+1^{B}$ observes $\left(\bar{d}_{k-1}^{B}, a\right)$ and follows her signal by Lemma B3.1. Since an analogous argument applies if $x_{k}=L$, with the roles of groups $A$ and $B$ reversed, Proposition 4.3 follows.

P4.4. Since neither group is in a cascade, by Lemma B4 we have that $\bar{d}_{k-1}^{A}$ and $\bar{d}_{k-1}^{B}$ are in $D_{k-1}^{b}$. Lemma B2 gives that the common player follows her signal $x_{k}$. Suppose $x_{k}=H$ so the common player chooses $a$. Then successive application of Lemma B3 gives that every subsequent player in both groups chooses $a$. Since an analogous argument applies if $x_{k}=L$, Proposition 4.4 follows.

Proof of Proposition 5. The proof is computational. We first consider the case of $i<k$, then we consider the case of $i \geq k$. Without loss, we take $i$ to be in group $A$.

Case (i), $i<k$ : Since $i$ is less than $k$, Lemmas B1 and B4 give that $i$ chooses $a$ if (i) $\bar{d}_{i-1}^{A} \in D_{i-1}^{b}$ and $x_{i}^{A}=H$ or (ii) $\bar{d}_{i-1}^{A} \in D_{i-1}^{A}$. Thus, Lemma B4 gives that the probability $i$ chooses $a$ given the state is $s$ is

$$
\operatorname{Prob}(i \text { chooses } a \mid s)= \begin{cases}\lambda^{\frac{i-2}{2}} P(H \mid s)+\frac{(P(H \mid s))^{2}}{1-\lambda}\left(1-\lambda^{\frac{i-2}{2}}\right) & \text { for } i \text { even } \\ \lambda^{\frac{i-1}{2}} P(H \mid s)+\frac{(P(H \mid s))^{2}}{1-\lambda}\left(1-\lambda^{\frac{i-1}{2}}\right) & \text { for } i \text { odd. }\end{cases}
$$

Thus, $i$ 's ex-ante payoff is

$$
w(i, k)=\operatorname{Prob}(i \text { chooses } a \mid \mathcal{H}) P(\mathcal{H})-\operatorname{Prob}(i \text { chooses } a \mid \mathcal{L}) P(\mathcal{L}),
$$


which simplifies to the stated expression. (We may ignore the times when $i$ chooses $r$ because choosing $r$ always gives zero.)

Case (ii), $i \geq k$ : We proceed by conditioning the expectation on the state and the type of cascades in both groups, as then Proposition 4 gives the decisions of all subsequent players. The exception is when both groups are in cascades on different actions. In these cases, we (successively) apply Lemma B3 to find the probability that a player chooses $a$. It is useful to differentiate these cases. Let $g \in\{A, B\}$ and let $c^{g} \in\{a, n, r\}$ denote the type of cascade in group $g$ before $k$ (e.g., $c^{g}=a \Longleftrightarrow \bar{d}_{k-1}^{g} \in D_{k-1}^{a}$ ). Let $C_{2}=\{(a, r),(r, a)\}$ be the set of cascades on different actions and $C_{1}=\{a, n, r\}^{2} \backslash C_{2}$ be the set of all other cascades.

Since the signals are conditionally independent given the state, we have that $c_{A}$ and $c_{B}$ are also conditionally independent given the state. Thus, $w(i, k)=g_{1}(i, k)+g_{2}(i, k)$, where

$$
\begin{aligned}
& g_{1}(i, k)=\sum_{\substack{s \in\{\mathcal{H}, \mathcal{L}\} \\
\left(c_{A}, c_{B}\right) \in C_{1}}} u(a, s) \operatorname{Prob}\left(i \text { chooses } a \mid s, c_{A}, c_{B}\right) \operatorname{Prob}\left(c_{A} \mid s\right) \operatorname{Prob}\left(c_{B} \mid s\right) P(s) \\
& g_{2}(i, k)=\sum_{\substack{s \in\{\mathcal{H}, \mathcal{L}\} \\
\left(c_{A}, c_{B}\right) \in C_{2}}} u(a, s) \operatorname{Prob}\left(i \text { chooses } a \mid s, c_{A}, c_{B}\right) \operatorname{Prob}\left(c_{A} \mid s\right) \operatorname{Prob}\left(c_{B} \mid s\right) P(s) .
\end{aligned}
$$

Here $\operatorname{Prob}\left(i\right.$ chooses $\left.a \mid s, c_{A}, c_{B}\right)$ is the probability that $i$ chooses $a$ in state $s$, and $\operatorname{Prob}(c \mid s)$ is given by Lemma B4.

First, we calculate $g_{1}$. Proposition 4 gives that if $\left(c_{A}, c_{B}\right) \in\{(a, a),(a, n),(n, a)\}$, then $i$ chooses $a$ with certainty, so $\operatorname{Prob}\left(i\right.$ chooses $\left.a \mid s, c_{A}, c_{B}\right)=1$ for both states $\mathcal{H}$ and $\mathcal{L}$. If $\left(c_{A}, c_{B}\right)=(n, n)$, then all players after $k$ choose $d_{k}$ with certainty. Since Lemma B2 gives $d_{k}=a$ if and only if $x_{k}=H$, we have $d_{k}=a$ with probability $P(H \mid s)$. Hence, $\operatorname{Prob}\left(i\right.$ chooses $\left.a \mid \mathcal{H}, c_{A}, c_{B}\right)=p$ and $\operatorname{Prob}\left(i\right.$ chooses $\left.a \mid L, c_{A}, c_{B}\right)=(1-p)$. Plugging these conditional probabilities into the expression for $g_{1}$ and simplifying gives

$$
g_{1}(i, k)=\frac{(2 p-1)\left(1-\lambda^{k}\right)}{2(1-\lambda)}
$$

Now we calculate $g_{2}$. Since the cascades are in $C_{2}$, we successively employ Lemma B3 to calculate the probability that $i$ chooses $a$. Table B3 gives the results of these computations. 
$\operatorname{Prob}\left(i\right.$ chooses $\left.a \mid s, c_{A}, c_{B}\right)$

\begin{tabular}{ccc}
\cline { 2 - 3 } Player Position & $\left(c_{A}, c_{B}\right)=(a, r)$ & $\left(c_{A}, c_{B}\right)=(r, a)$ \\
\hline$k$ & $P(H \mid s)$ & $P(H \mid s)$ \\
\hline$k+1$ & $P(H \mid s)+P(L \mid s) P(H \mid s)$ & $P(H \mid s)^{2}$ \\
\hline$k+2$ & $P(H \mid s)+P(L \mid s) P(H \mid s)^{2}$ & $P(H \mid s)^{2}+P(L \mid s) P(H \mid s)^{2}$ \\
\hline$i>k+2$ and $i$ odd & $P(H \mid s)$ & $P(H \mid s)^{2}$ \\
& $+P(L \mid s) P(H \mid s)^{2} \lambda^{\frac{i-k-2}{2}}$ & $+P(L \mid s) P(H \mid s)^{2} \lambda^{\frac{i-k-2}{2}}$ \\
& $+P(L \mid s) P(H \mid s)^{3} \Lambda(i-k-2)$ & $+P(L \mid s) P(H \mid s)^{3} \Lambda(i-k-2)$ \\
\hline & $P(H \mid s)$ & $P(H \mid s)^{2}$ \\
& $+P(L \mid s) P(H \mid s)^{2} \lambda^{\frac{i-k-3}{2}}$ & $+P(L \mid s) P(H \mid s)^{2} \lambda^{\frac{i-k-3}{2}}$ \\
& $+P(L \mid s) P(H \mid s)^{3} \Lambda(i-k-3)$ & $+P(L \mid s) P(H \mid s)^{3} \Lambda(i-k-3)$ \\
\hline
\end{tabular}

Table B3: Probability Player $i$ Chooses $a$

We illustrate the construction of the bottom-left cell: $\left(c_{A}, c_{B}\right)=(a, r), i>k+2$, and $i$ even. We begin by noting that successive application of Lemma B3 shows that the only histories $i$ observes in equilibrium are those listed in Table B2(c). Thus, $i$ chooses $a$ if (i) $d_{k}=d_{k+1}^{A}=\ldots=d_{i-1}^{A}=a$ (i.e., a cascade on $a$ immediately after $k$ ), (ii) $d_{k}=r, d_{k+1}^{A}=a$, $\left(d_{k+2}^{A}, \ldots, d_{i-1}^{A}\right) \in D_{i-k-2}^{b}$ (i.e., no cascade after $k$ ), and $x_{i}^{A}=H$, or (iii) $d_{k}=r, d_{k+1}^{A}=a$, and $\left(d_{k+2}^{A}, \ldots, d_{i-1}^{A}\right) \in D_{i-k-2}^{a}$ (i.e., a cascade on $a$ at some point after $k$ ). Since Lemma B2 gives $d_{k}=a \Longleftrightarrow x_{k}=H$, the (conditional) probability of (i) is $P(H \mid s)$. As to the probability of (ii): Lemmas B2 and B3 give that $x_{k}=L, x_{k+1}^{A}=H$, and $x_{i}=H$. In addition, Lemma B3 gives that players $k+2^{A}$ to $i-1^{A}$ follow their signals, which implies that the probability $\left(d_{k+2}^{A}, \ldots, d_{i-1}^{A}\right) \in D_{i-k-2}^{b}$ is $\lambda^{\frac{i-k-3}{2}}$. Thus, the probability of (ii) is $P(L \mid s) P(H \mid s)^{2} \lambda^{\frac{i-k-3}{2}}$. As to the probability of (iii): We have $x_{k}=L$ and $x_{k+1}^{A}=H$. Since $\left(d_{k+2}^{A}, \ldots, d_{i-1}^{A}\right) \in D_{i-k-2}^{a}$, there is an odd $\theta<i-1$ such that $\left(d_{k+2}^{A}, \ldots, d_{\theta}^{A}\right)$ is balanced and $d_{\theta}^{A}=d_{\theta+1}^{A}=\cdots=d_{i-1}^{A}=a$. By Lemma B3, players $k+2^{A}$ to $\theta+1^{A}$ follow their own signal, while players $\theta^{A}+2$ to $i-1^{A}$ are in a cascade on $a$. This implies the probability $\left(d_{k+2}^{A}, \ldots, d_{i-1}^{A}\right) \in D_{i-k-2}^{a}$ is $P(H \mid s)^{2} \Lambda(i-k-3)$ (because there are $\frac{i-k-3}{2}$ places to put $\theta$ ). Thus, the probability of (iii) is $P(L \mid s) P(H \mid s)^{3} \Lambda(i-k-3)$. Summing the probabilities of (i), (ii), and (iii) gives the cell. 
Plugging the values from Table B3 into the expression for $g_{2}$ and simplifying gives

$$
g_{2}(i, k)=(2 p-1)\left(\frac{\lambda}{2} \Lambda(k-1)\right)^{2} \begin{cases}1 & \text { if } i=k \text { or } i=k+1 \\ \left(1+\frac{\lambda}{2} \Lambda(i-k)\right) & \text { if } i \geq k+2 .\end{cases}
$$

Thus, the display equation of the proposition is true.

Proof of Proposition 6. In a basic cascade, the players' asymptotic payoff is

$$
\lim _{i \rightarrow \infty} w^{B}(i)=\lim _{i \rightarrow \infty} \frac{2 p-1}{2} \Lambda(i)=\frac{2 p-1}{2(1-\lambda)} .
$$

In an interacting cascade, the payoff of the common player is

$$
w(k, k)=\frac{2 p-1}{2(1-\lambda)}\left[1-\lambda^{k}+\frac{1}{2(1-\lambda)}\left[\lambda\left(1-\lambda^{\frac{k-1}{2}}\right)\right]^{2}\right] .
$$

Hence, $w(k, k) \geq \lim _{i \rightarrow \infty} w^{B}(i)$ if and only if

$$
1-\lambda^{k}+\frac{1}{2(1-\lambda)}\left[\lambda\left(1-\lambda^{\frac{k-1}{2}}\right)\right]^{2} \geq 1,
$$

which simplifies to

$$
(1-\lambda)\left(1-3 \lambda^{k-2}\right)+\lambda^{-2}\left(\lambda^{\frac{3}{2}}-\lambda^{\frac{k}{2}}\right)^{2} \geq 0 .
$$

Since $p \in(1 / 2,1)$, then $\lambda=2 p(1-p) \in(0,1 / 2)$. If $k \geq 5$, then $1-3 \lambda^{k-2}>0$ and $\lambda^{\frac{3}{2}}-\lambda^{\frac{k}{2}}>0$, and hence $w(k, k)>\lim _{i \rightarrow \infty} w^{B}(i)$. While if $k=3$, then $\lambda^{\frac{3}{2}}-\lambda^{\frac{k}{2}}=0$ and $1-3 \lambda \geq 0$ if $\lambda \leq 1 / 3$ (i.e., $p \geq \frac{1}{6} \sqrt{3}+\frac{1}{2}$ ), and hence $w(k, k) \geq \lim _{i \rightarrow \infty} w^{B}(i)$. Since $w^{B}(j)<\lim _{i \rightarrow \infty} w^{B}(i)$ for each $j$, then $w(k, k)>w^{B}(i)$ for all $i$ if either $k \geq 5$ or $k=3$ and $p \geq \frac{1}{6} \sqrt{3}+\frac{1}{2}$.

If $k=3$ and $p<\frac{1}{6} \sqrt{3}+\frac{1}{2}$ then $w(k, k)<\lim _{i \rightarrow \infty} w^{B}(i)$, so there is some $i$ such that $w(k, k)<w^{B}(i)$.

Proof of Proposition 7. We prove the result by establishing that the gain from moving the common player exceeds the loss when $p$ is large. Since players in positions $1, \ldots, k-3$ in both groups are unaffected by the move, we only need to show that the (i) the minimum gain for players $k-2^{A}, k-1^{A}, k-2^{B}$, and $k-1^{B}$ exceeds the (ii) maximum loss for the common player and players $k+1^{A}, k+1^{B}, \ldots, N^{A}$, and $N^{B}$. With this in mind, we proceed by constructing a (i') lower bound on the gain and (ii') an upper bound on the loss. We then establish that (i') is strictly greater than (ii') when $p$ is sufficiently close to 1 . The desired result follows. 
First, we compute a lower bound on the gain for players $k-2^{A}, k-1^{A}, k-2^{B}$, and $k-1^{B}$. Table B4 computes the gain to each of these players.

\begin{tabular}{cc} 
Player & Gain \\
\hline$k-2$ & $w(k-1, k-2)-w(k-2, k)=\frac{2 p-1}{2} \lambda^{\frac{k-1}{2}} \Lambda(k-3)+(2 p-1)\left(\Lambda(k-3) \frac{\lambda}{2}\right)^{2}$ \\
\hline$k-1$ & $w(k, k-2)-w(k-1, k)=\frac{2 p-1}{2} \lambda^{\frac{k-1}{2}} \Lambda(k-3)+(2 p-1)\left(\Lambda(k-3) \frac{\lambda}{2}\right)^{2}\left(1+\frac{\lambda}{2}\right)$ \\
\hline
\end{tabular}

Table B4: Gain to Players $k-2$ and $k-1$

It is clear from the table that the total gain to all four players is at least

$$
G(p)=2(2 p-1) \lambda^{\frac{k-1}{2}} \Lambda(k-3)
$$

Second, we compute an upper bound on the loss for the common player and subsequent players in both groups. Table B5 computes the loss to each of these players.

\begin{tabular}{cc}
\multicolumn{1}{c}{ Player } & Loss \\
\hline Common Player & $w(k, k)-w(k-2, k-2)=$ \\
& $\frac{2 p-1}{2} \lambda^{k-2} \Lambda(4)+(2 p-1)\left(\frac{\lambda}{2}\right)^{2}\left(\Lambda(k-1)^{2}-\Lambda(k-3)^{2}\right)$ \\
\hline$k+1$ & $w(k+1, k)-w(k+1, k-2)=$ \\
& $\frac{2 p-1}{2} \lambda^{k-2} \Lambda(4)+(2 p-1)\left(\frac{\lambda}{2}\right)^{2}\left(\Lambda(k-1)^{2}-\Lambda(k-3)^{2}\left(1+\frac{\lambda}{2}\right)\right)$ \\
\hline$i \geq k+2$ and $i$ odd & $w(i, k)-w(i, k-2)=\frac{2 p-1}{2} \lambda^{k-2} \Lambda(4)+(2 p-1)\left(\frac{\lambda}{2}\right)^{2}[$ \\
$i \geq k+2$ and $i$ even & $\left.(k-1)^{2}\left(1+\frac{\lambda}{2} \Lambda(i-k)\right)-\Lambda(k-3)^{2}\left(1+\frac{\lambda}{2} \Lambda(i-k+2)\right)\right]$ \\
\hline
\end{tabular}

Table B5: Loss to Common Player and Subsequent Players

A bit of algebra shows that every row of Table B5 is less than or equal

$$
T(p)=\frac{2 p-1}{2} \lambda^{k-2} \Lambda(4)+2(2 p-1)\left(\frac{\lambda}{2}\right)^{2}\left(\Lambda(k-1)^{2}-\Lambda(k-3)^{2}\right) .
$$

Consequently, the loss to the common player and all subsequent players is at most

$$
L(p)=2\left(N-k+\frac{1}{2}\right) T(p) .
$$


We now establish that $G(p) \geq L(p)$ for $p$ sufficiently close to 1 , this then implies that the move of the common player is surplus improving. To do this, consider the related inequality

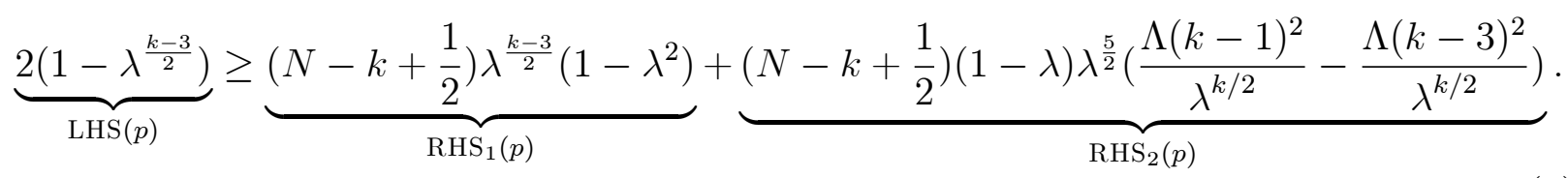

Observe that $G(p)=\frac{2 p-1}{(1-\lambda)} \lambda^{\frac{k-1}{2}} \operatorname{LHS}(p)$ and that $L(p)=\frac{2 p-1}{1-\lambda} \lambda^{\frac{k-1}{2}}\left(\operatorname{RHS}_{1}(p)+\operatorname{RHS}_{2}(p)\right)$. Notice that (i) $\operatorname{LHS}(1)=2$, (ii) $\operatorname{RHS}_{1}(1)=0$, and (iii) $\operatorname{RHS}_{2}(p)=0$. The first and second equalities are obvious. The third equality follows from the fact that $(1-\lambda) \lambda^{\frac{5}{2}\left(\frac{\Lambda(k-1)^{2}}{\lambda^{k / 2}}-\right.}$ $\left.\frac{\Lambda(k-3)^{2}}{\lambda^{k / 2}}\right)=2 \lambda-\lambda^{\frac{k-1}{2}}(1+\lambda)$, which equals 0 when $p=1$ since $k>1$. It follows that (2) is true with strict inequality when $p=1$. Since both sides of (2) are continuous in $p$, there is a $p_{k}$, with $\frac{1}{2}<p_{k}<1$, such that (2) is true for all $p \geq p_{k}$. At every $p \in\left[p_{k}, 1\right)$, we have that $\frac{2 p-1}{1-\lambda} \lambda^{\frac{k-1}{2}}>0$. Multiplying both sides of $(2)$ by $\frac{2 p-1}{1-\lambda} \lambda^{\frac{k-1}{2}}$ establishes that that $G(p) \geq L(p)$, as desired. 


\section{Appendix C: Online Supplement}

In this appendix, we prove Lemmas B1 to B4. First, we define balanced and unbalanced signal profiles and establish several preliminary lemmas.

A signal profile $\bar{x}_{i}=\left(x_{1}, \ldots, x_{i}\right)$ is balanced if, for every odd integer $j<i$, we have that $x_{j} \neq x_{j+1}$, and is unbalanced otherwise. Equivalently, a signal profile is balanced if the running difference between the total number of $H$ signals and the total number of $L$ signals never exceeds 1 . The null signal profile and every singleton signal profile are trivially balanced.

If a signal profile $\bar{x}_{i}$ is unbalanced then there is an odd integer $j<i$ such that $x_{j}=x_{j+1}$. Let $\theta$ denote the smallest such $j$. We say that an unbalance signal profile is unbalanced on $H$ if $x_{\theta+1}=H$ and is unbalanced on $L$ if $x_{\theta+1}=L$. As Lemmas C2 and C3 will make clear, $\theta+1$ is the last player who in equilibrium will follow her own signal, i.e., chooses $a$ given signal $H$ and chooses $L$ given signal $L{ }^{10}$

Let $X_{i}=\{H, L\}^{i}$. Let $X_{i}^{b}$ denote the set of balanced $i$-length signal profiles, let $X_{i}^{H}$ denote the set of $i$-length signal profiles that are unbalanced on $H$, and let $X_{i}^{L}$ denote the set of $i$-length signal profiles that are unbalanced on $L$. The sets $X_{i}^{b}, X_{i}^{H}$, and $X_{i}^{L}$ partition $X_{i}$.

For $T \subset X_{i}$, we write

$$
\operatorname{Prob}(T \mid s)=\Sigma_{\bar{x}_{i} \in T} P\left(x_{1} \mid s\right) \cdots P\left(x_{i} \mid s\right)
$$

for the probability the realized signal profile is in $T$ given state $s$.

Lemma C1. For $i \geq 2$, let $\bar{x}_{i}$ be a signal profile. Then, for $s \in\{\mathcal{H}, \mathcal{L}\}$,

\footnotetext{
${ }^{10}$ Observe that if a signal profile is unbalanced on $H(L)$, then the elements of the profile after $j$ may be arbitrary. In contrast, the elements of a history that is unbalanced on $a(r)$ are not arbitrary after $j$.
} 


$$
\begin{aligned}
\operatorname{Prob}\left(X_{i}^{b} \mid s\right) & = \begin{cases}\lambda^{\frac{i}{2}} & \text { for } i \text { even } \\
\lambda^{\frac{i-1}{2}} & \text { for } i \text { odd, }\end{cases} \\
\operatorname{Prob}\left(X_{i}^{H} \mid s\right) & = \begin{cases}P(H \mid s)^{2} \Lambda(i) & \text { for } i \text { even } \\
P(H \mid s)^{2} \Lambda(i-1) & \text { for } i \text { odd },\end{cases} \\
\operatorname{Prob}\left(X_{i}^{L} \mid s\right) & = \begin{cases}P(L \mid s)^{2} \Lambda(i) & \text { for } i \text { even } \\
P(L \mid s)^{2} \Lambda(i-1) & \text { for } i \text { odd. }\end{cases}
\end{aligned}
$$

Proof. The proof is a simple computation and is omitted.

Proof of Lemma B1. By induction. Let $\left(\sigma^{*}, \phi^{*}\right)$ be an equilibrium. Consider player 1 with signal $x_{1}$. Her belief is

$$
\begin{aligned}
\phi_{i}^{*}\left(\bar{d}_{0}, x_{1}\right) & =\frac{P(\mathcal{H}) P\left(x_{1} \mid \mathcal{H}\right) P_{\sigma^{*}}\left(\bar{d}_{0} \mid \mathcal{H}\right)}{\sum_{s \in\{\mathcal{H}, \mathcal{L}\}} P(s) P\left(x_{1} \mid s\right) P_{\sigma^{*}}\left(\bar{d}_{0} \mid s\right)} \\
& =\left\{\begin{array}{cl}
p & \text { if } x_{1}=H \\
1-p & \text { if } x_{1}=L .
\end{array}\right.
\end{aligned}
$$

Since $p>1 / 2$, sequential rationality implies $\sigma_{1}^{*}\left(\bar{d}_{0}, H\right)=a$ and $\sigma_{1}^{*}\left(\bar{d}_{0}, L\right)=r$. Since $\bar{d}_{0} \in D_{0}^{b}$, Lemma B.1 holds for $i=1$.

Assume Lemma B.1 holds for players 1 to $i-1$. We show it holds for $i$. Let $\bar{d}_{i-1}$ be the history observed by $i$. By the induction hypothesis, $\bar{d}_{i-1} \in D_{i-1}^{a} \cup D_{i-1}^{b} \cup D_{i-1}^{r}$, so there are three cases.

Case (i): If $\bar{d}_{i-1} \in D_{i-1}^{b}$ then the induction hypothesis implies that each player prior to $i$ has followed her own signal. Thus

$$
P_{\sigma^{*}}\left(d_{1}, \ldots, d_{i-1} \mid s\right)=\prod_{j=1}^{i-1} P\left(x_{j} \mid s\right) \text { where } x_{j}=\left\{\begin{array}{cc}
H & \text { if } d_{j}=a \\
L & \text { if } d_{j}=r .
\end{array}\right.
$$

If $i$ is odd then $P_{\sigma^{*}}\left(d_{1}, \ldots, d_{i-1} \mid s\right)=(\lambda / 2)^{\frac{i-1}{2}}$, and if $i$ is even then $P_{\sigma^{*}}\left(d_{1}, \ldots, d_{i-1} \mid s\right)=$ $(\lambda / 2)^{\frac{i-2}{2}} P\left(x_{i-1} \mid s\right)$. Thus player $i$ 's belief given signal $x_{i}$ is

$$
\phi_{i}^{*}\left(d_{1}, \ldots, d_{i-1}, x_{i}\right)=\frac{P(\mathcal{H}) P\left(x_{i} \mid \mathcal{H}\right) P_{\sigma^{*}}\left(d_{1}, \ldots, d_{i-1} \mid \mathcal{H}\right)}{\sum_{s \in\{\mathcal{H}, \mathcal{L}\}} P(s) P\left(x_{i} \mid s\right) P_{\sigma^{*}}\left(d_{1}, \ldots, d_{i-1} \mid s\right)}
$$


If $i$ is odd, then

$$
\begin{aligned}
\phi_{i}^{*}\left(d_{1}, \ldots, d_{i-1}, x_{i}\right) & =\frac{P(\mathcal{H}) P\left(x_{i} \mid \mathcal{H}\right)(\lambda / 2)^{\frac{i-1}{2}}}{\sum_{s \in\{\mathcal{H}, \mathcal{L}\}} P(s) P\left(x_{i} \mid s\right)(\lambda / 2)^{\frac{i-1}{2}}}=\frac{P(\mathcal{H}) P\left(x_{i} \mid \mathcal{H}\right)}{\sum_{s \in\{\mathcal{H}, \mathcal{L}\}} P(s) P\left(x_{i} \mid s\right)} \\
& =\left\{\begin{array}{cl}
p & \text { if } x_{i}=H \\
1-p & \text { if } x_{i}=L .
\end{array}\right.
\end{aligned}
$$

Thus sequential rationality implies $\sigma_{i}^{*}\left(\bar{d}_{i-1}, H\right)=a$ and $\sigma_{i}^{*}\left(\bar{d}_{i-1}, L\right)=r$.

If $i$ is even, then

$\phi_{i}^{*}\left(d_{1}, \ldots, d_{i-1}, x_{i}\right)=\frac{P(\mathcal{H}) P\left(x_{i} \mid \mathcal{H}\right)(\lambda / 2)^{\frac{i-2}{2}} P\left(x_{i-1} \mid \mathcal{H}\right)}{\sum_{s \in\{\mathcal{H}, \mathcal{L}\}} P(s) P\left(x_{i} \mid s\right)(\lambda / 2)^{\frac{i-2}{2}} P\left(x_{i-1} \mid s\right)}=\frac{P(\mathcal{H}) P\left(x_{i} \mid \mathcal{H}\right) P\left(x_{i-1} \mid \mathcal{H}\right)}{\sum_{s \in\{\mathcal{H}, \mathcal{L}\}} P(s) P\left(x_{i} \mid s\right) P\left(x_{i-1} \mid s\right)}$.

If $\left(x_{i-1}, x_{i}\right)=(L, H)$ or $(H, H)$ then it is readily verified that $\phi_{i}^{*}\left(d_{1}, \ldots, d_{i-1}, x_{i}\right) \geq 1 / 2$, while if $\left(x_{i-1}, x_{i}\right)=(H, L)$ or $(L, L)$ then $\phi_{i}^{*}\left(d_{1}, \ldots, d_{i-1}, x_{i}\right) \leq 1 / 2$. Thus sequential rationality implies $\sigma_{i}^{*}\left(\bar{d}_{i-1}, H\right)=a$ and $\sigma_{i}^{*}\left(\bar{d}_{i-1}, L\right)=r$.

Case (ii): Suppose $\bar{d}_{i-1} \in D_{i-1}^{a}$. There is an odd integer $j<i-1$ such that $d_{j}=d_{j+1}=a$. Let $\theta$ denote the smallest such $j$. Note that $\left(d_{1}, \ldots, d_{\theta}\right)$ is balanced. By the induction hypothesis (a) players 1 through $\theta+1$ follow their own signals and (b) players $\theta+2$ through $i-1$ take the same action as player $\theta+1$ regardless of their own signals. Thus

$$
P_{\sigma^{*}}\left(d_{1}, \ldots, d_{i-1} \mid s\right)=\prod_{j=1}^{\theta+1} P\left(x_{j} \mid s\right) \text { where } x_{j}=\left\{\begin{aligned}
H & \text { if } d_{j}=a \\
L & \text { if } d_{j}=r
\end{aligned}\right.
$$

Since $\left(d_{1}, \ldots, d_{\theta}\right)$ is balanced and $d_{\theta}=d_{\theta+1}=a$ then

$$
P_{\sigma^{*}}\left(d_{1}, \ldots, d_{i-1} \mid s\right)=(\lambda / 2)^{\frac{\theta-1}{2}} P\left(x_{\theta} \mid s\right) P\left(x_{\theta+1} \mid s\right)=(\lambda / 2)^{\frac{\theta-1}{2}} P(H \mid s)^{2} .
$$

Hence

$$
\phi_{i}^{*}\left(d_{1}, \ldots, d_{i-1}, x_{i}\right)=\frac{P(\mathcal{H}) P\left(x_{i} \mid \mathcal{H}\right)(\lambda / 2)^{\frac{\theta-1}{2}} P(H \mid \mathcal{H})^{2}}{\sum_{s \in\{\mathcal{H}, \mathcal{L}\}} P(s) P\left(x_{i} \mid s\right)(\lambda / 2)^{\frac{\theta-1}{2}} P(H \mid s)^{2}}=\frac{P(\mathcal{H}) P\left(x_{i} \mid \mathcal{H}\right) P(H \mid \mathcal{H})^{2}}{\sum_{s \in\{\mathcal{H}, \mathcal{L}\}} P(s) P\left(x_{i} \mid s\right) P(H \mid s)^{2}}
$$

It is readily verified that $\phi_{i}^{*}\left(d_{1}, \ldots, d_{i-1}, x_{i}\right)>1 / 2$ for $x_{i} \in\{H, L\}$. Thus sequential rationality implies $\sigma_{i}^{*}\left(\bar{d}_{i-1}, x_{i}\right)=a$.

Case (iii): When $\bar{d}_{i-1} \in D_{i-1}^{r}$, a symmetric argument to Case (ii) gives that $i$ chooses $r$.

Observe that if player $i$ observes a history $\bar{d}_{i-1}$ that's not given by a row of Table B1, then $\bar{d}_{i-1}$ is not an equilibrium path history, so $i$ 's belief and strategy are arbitrary. 
Proof of Lemma B4. This result is an immediate corollary of Lemmas B1 and C1.

Proof of Lemma B2. Let $\left(\bar{d}_{k-1}^{A}, \bar{d}_{k-1}^{B}\right) \in\left(D_{k-1}^{b} \cup D_{k-1}^{a} \cup D_{k-1}^{r}\right)^{2}$. If $\bar{d}_{k-1}^{g} \in D_{k-1}^{b}$ then $P_{\sigma^{*}}\left(\bar{d}_{k-1}^{g} \mid s\right)=\lambda^{\frac{k-1}{2}}$. If $\bar{d}_{k-1}^{g} \in D_{k-1}^{a}$ then $P_{\sigma^{*}}\left(\bar{d}_{k-1}^{g} \mid s\right)=(\lambda / 2)^{\frac{\theta-1}{2}} p(H \mid s)^{2}$, and if $\bar{d}_{k-1}^{g} \in D_{k-1}^{r}$ then $P_{\sigma^{*}}\left(\bar{d}_{k-1}^{g} \mid s\right)=(\lambda / 2)^{\frac{\theta-1}{2}} p(L \mid s)^{2}$, where $\theta$ denotes the smallest odd integer $j<i-1$ such that $d_{j}=d_{j+1}=a(=r)$. Thus, $k$ 's belief is

$$
\phi_{k}^{*}\left(\bar{d}_{k-1}^{A}, \bar{d}_{k-1}^{B}, x_{k}\right)=\frac{P(\mathcal{H}) P\left(x_{k} \mid \mathcal{H}\right) P_{\sigma^{*}}\left(\bar{d}_{k-1}^{A} \mid \mathcal{H}\right) P_{\sigma^{*}}\left(\bar{d}_{k-1}^{B} \mid \mathcal{H}\right)}{\sum_{s \in\{\mathcal{H}, \mathcal{L}\}} P(s) P\left(x_{k} \mid s\right) P_{\sigma^{*}}\left(\bar{d}_{k-1}^{A} \mid s\right) P_{\sigma^{*}}\left(\bar{d}_{k-1}^{B} \mid s\right)} .
$$

Using this expression, the previous facts, and a bit of algebra, we get Table C1, a description of when $\phi_{k}^{*}(\cdot)$ is greater than, less than, or equal to $1 / 2$.

\begin{tabular}{cccc} 
& & \multicolumn{2}{c}{$x_{k}$} \\
\cline { 2 - 4 } $\bar{d}_{k-1}^{A}$ & $\bar{d}_{k-1}^{B}$ & $H$ & $L$ \\
\hline \multirow{3}{*}{$D_{k-1}^{a}$} & $D_{k-1}^{a}$ & $\phi_{k}^{*}>\frac{1}{2}$ & $\phi_{k}^{*}>\frac{1}{2}$ \\
\cline { 2 - 4 } & $D_{k-1}^{b}$ & $\phi_{k}^{*}>\frac{1}{2}$ & $\phi_{k}^{*}>\frac{1}{2}$ \\
\cline { 2 - 4 } & $D_{k-1}^{r}$ & $\phi_{k}^{*}>\frac{1}{2}$ & $\phi_{k}^{*}<\frac{1}{2}$ \\
\hline \multirow{3}{*}{$D_{k-1}^{b}$} & $D_{k-1}^{a}$ & $\phi_{k}^{*}>\frac{1}{2}$ & $\phi_{k}^{*}>\frac{1}{2}$ \\
\cline { 2 - 4 } & $D_{k-1}^{b}$ & $\phi_{k}^{*}>\frac{1}{2}$ & $\phi_{k}^{*}<\frac{1}{2}$ \\
\cline { 2 - 4 } & $D_{k-1}^{r}$ & $\phi_{k}^{*}<\frac{1}{2}$ & $\phi_{k}^{*}<\frac{1}{2}$ \\
\hline \multirow{3}{*}{$D_{k-1}^{r}$} & $D_{k-1}^{a}$ & $\phi_{k}^{*}>\frac{1}{2}$ & $\phi_{k}^{*}<\frac{1}{2}$ \\
\cline { 2 - 4 } & $D_{k-1}^{b}$ & $\phi_{k}^{*}<\frac{1}{2}$ & $\phi_{k}^{*}<\frac{1}{2}$ \\
\cline { 2 - 4 } & $D_{k-1}^{r}$ & $\phi_{k}^{*}<\frac{1}{2}$ & $\phi_{k}^{*}<\frac{1}{2}$ \\
\hline
\end{tabular}

Table C1: The Beliefs of the Common Player

Table B1(b) records the sequentially rational action for each cell of Table C1.

If $\left(\bar{d}_{k-1}^{A}, \bar{d}_{k-1}^{B}\right) \notin\left(D_{k-1}^{b} \cup D_{k-1}^{a} \cup D_{k-1}^{r}\right)^{2}$, then $\left(\bar{d}_{k-1}^{A}, \bar{d}_{k-1}^{B}\right)$ is not an equilibrium history by Lemma B1. The common player's belief and strategy are arbitrary.

\section{After the Common Player}

We prove each part of Lemma B3 separately. Player $k+1$ observes a history in $\left\{D_{k-1}^{a} \cup\right.$ $\left.D_{k-1}^{b} \cup D_{k-1}^{r}\right\} \times\{a, r\}$ by Lemmas B1 and B2. To compute her belief, we need the following lemma that gives the equilibrium probability distribution over this set. 
Lemma C2. Let $g \in\{A, B\}$ and $\left(\bar{d}_{k-1}^{g}, d_{k}\right)$ be a history with $\bar{d}_{k-1}^{g} \in D_{k-1}^{a} \cup D_{k-1}^{b} \cup D_{k-1}^{r}$. Then $P_{\sigma^{*}}^{g}\left(\bar{d}_{k-1}^{g}, d_{k} \mid s\right)$ is given by Table C2 for $s \in\{\mathcal{H}, \mathcal{L}\}$.

\begin{tabular}{ccc} 
& \multicolumn{2}{c}{$P_{\sigma^{*}}^{g}\left(\bar{d}_{k-1}^{g}, d_{k} \mid s\right)$} \\
\cline { 2 - 3 } $\bar{d}_{k-1}^{g}$ & $d_{k}=a$ & $d_{k}=r$ \\
\hline$D_{k-1}^{a}$ & $P_{\sigma^{*}}^{g}\left(\bar{d}_{k-1}^{g} \mid s\right)\left(1-P(L \mid s)^{3} \Lambda(k-1)\right)$ & $P_{\sigma^{*}}^{g}\left(\bar{d}_{k-1}^{g} \mid s\right) P(L \mid s)^{3} \Lambda(k-1)$ \\
\hline$D_{k-1}^{b}$ & $P_{\sigma^{*}}^{g}\left(\bar{d}_{k-1}^{g} \mid s\right) P(H \mid s) \lambda^{\frac{k-1}{2}}$ & $P_{\sigma^{*}}^{g}\left(\bar{d}_{k-1}^{g} \mid s\right) P(L \mid s) \lambda^{\frac{k-1}{2}}$ \\
& $+P_{\sigma^{*}}^{g}\left(\bar{d}_{k-1}^{g} \mid s\right) P(H \mid s)^{2} \Lambda(k-1)$ & $+P_{\sigma^{*}}^{g}\left(\bar{d}_{k-1}^{g} \mid s\right) P(L \mid s)^{2} \Lambda(k-1)$ \\
\hline$D_{k-1}^{r}$ & $P_{\sigma^{*}}^{g}\left(\bar{d}_{k-1}^{g} \mid s\right) P(H \mid s)^{3} \Lambda(k-1)$ & $P_{\sigma^{*}}^{g}\left(\bar{d}_{k-1}^{g} \mid s\right)\left(1-P(H \mid s)^{3} \Lambda(k-1)\right)$ \\
\hline
\end{tabular}

Table C2: Value of $P_{\sigma^{*}}^{g}\left(\bar{d}_{k-1}^{g}, d_{k} \mid s\right)$

Proof. This lemma is computational. Without loss let $g=A$. Given $\left(\bar{d}_{k-1}^{A}, d_{k}\right)$, Lemma B2 gives the possible pairings $\left(\bar{d}_{k-1}^{B}, x_{k}\right)$ for which player $k$ chooses $d_{k}$. Given these, we apply Lemma B4 to compute $P_{\sigma^{*}}^{A}\left(\bar{d}_{k-1}^{A}, d_{k} \mid s\right)$. We illustrate the calculation of the top-right cell of Table C2, i.e., when $\bar{d}_{k-1}^{A} \in D_{k-1}^{a}$ and $d_{k}=r$. The common player then chooses $r$ when $\bar{d}_{k-1}^{B} \in D_{k-1}^{r}$ and $x_{k}=L$ by Lemma B2. Hence,

$$
\begin{aligned}
P_{\sigma^{*}}^{A}\left(\bar{d}_{k-1}^{A}, r \mid s\right) & =P_{\sigma^{*}}^{A}\left(\bar{d}_{k-1}^{A} \mid s\right) P(L \mid s) \operatorname{Prob}\left(D_{k-1}^{r} \mid s\right) \\
& =P_{\sigma^{*}}^{A}\left(\bar{d}_{k-1}^{A} \mid s\right) P(L \mid s)^{3} \Lambda(k-1)
\end{aligned}
$$

where the second equality follows from Lemma B4.

Proof of Lemma B3.1. Without loss we consider player $k+1^{A}$. Let $\left(\bar{d}_{k-1}^{A}, d_{k}\right)=$ $\left(d_{1}^{A}, \ldots, d_{k-1}^{A}, d_{k}\right) \in\left\{D_{k-1}^{a} \cup D_{k-1}^{b} \cup D_{k-1}^{r}\right\} \times\{a, r\}$. If $\bar{d}_{k-1}^{A} \in D_{k-1}^{a}$ or $\bar{d}_{k-1}^{A} \in D_{k-1}^{r}$, then there is an odd integer $j<k-1$ such that $d_{j}^{A}=d_{j+1}^{A}$. Let $\theta$ denote the smallest such $j$. Note that $\left(d_{1}^{A}, \ldots, d_{\theta}^{A}\right)$ is balanced. If $\bar{d}_{k-1}^{A} \in D_{k-1}^{b}$, let $\theta=k-2$.

We proceed by deriving the belief of player $k+1^{A}$. Recall that

$$
\phi_{k+1}^{A *}\left(\bar{d}_{k-1}^{A}, d_{k}, x_{k+1}^{A}\right)=\frac{P(\mathcal{H}) P\left(x_{k+1}^{A} \mid \mathcal{H}\right) P_{\sigma^{*}}^{A}\left(\bar{d}_{k-1}^{A}, d_{k} \mid \mathcal{H}\right)}{\sum_{s \in\{\mathcal{H}, \mathcal{L}\}} P(s) P\left(x_{k+1}^{A} \mid s\right) P_{\sigma^{*}}^{A}\left(\bar{d}_{k-1}^{A}, d_{k} \mid s\right)} .
$$

Lemma B1 implies that players $1^{A}$ to $\theta+1^{A}$ follow their own signals, while players $\theta+2^{A}$ to $k-1^{A}$ are in a cascade. Thus, $x_{i}^{A}=H$ if $d_{i}^{A}=a$ and $x_{i}^{A}=L$ if $d_{i}^{A}=r$ for all $i \in\{1, \ldots, \theta+1\}$. Hence, $P_{\sigma^{*}}^{A}\left(\bar{d}_{k-1}^{A} \mid s\right)=P\left(x_{\theta}^{A} \mid s\right) P\left(x_{\theta+1}^{A} \mid s\right)(\lambda / 2)^{\frac{\theta-1}{2}}$. Thus we use Lemma C2 
to write the following table that describes when the belief of player $k+1^{A}$ is greater than, equal to, or less than $\frac{1}{2}$.

\begin{tabular}{cccc} 
& & \multicolumn{2}{c}{$\sigma_{k+1}^{g *}\left(\bar{d}_{k}^{g}, x_{k+1}^{g}\right)$} \\
\cline { 2 - 4 } $\bar{d}_{k-1}^{g}$ & $d_{k}$ & $H$ & $L$ \\
\hline$D_{k-1}^{a}$ & $a$ & $\phi_{k+1}^{A *}>\frac{1}{2}$ & $\phi_{k+1}^{A *}>\frac{1}{2}$ \\
\cline { 2 - 4 } & $r$ & $\phi_{k+1}^{A *}=\frac{1}{2}$ & $\phi_{k+1}^{A *}<\frac{1}{2}$ \\
\hline$D_{k-1}^{b}$ & $a$ & $\phi_{k+1}^{A *}>\frac{1}{2}$ & $\phi_{k+1}^{A *}>\frac{1}{2}$ \\
\cline { 2 - 4 } & $r$ & $\phi_{k+1}^{A *}<\frac{1}{2}$ & $\phi_{k+1}^{A *}<\frac{1}{2}$ \\
\hline$D_{k-1}^{r}$ & $a$ & $\phi_{k+1}^{A *}>\frac{1}{2}$ & $\phi_{k+1}^{A *}=\frac{1}{2}$ \\
\cline { 2 - 4 } & $r$ & $\phi_{k+1}^{A *}<\frac{1}{2}$ & $\phi_{k+1}^{A *}<\frac{1}{2}$ \\
\hline
\end{tabular}

Table C3: When $\phi_{k+1}^{A *}(\cdot)$ is $>,=$, and $<1 / 2$

We illustrate the calculation of the top-left cell of Table C3, i.e., when that $\bar{d}_{k-1}^{A} \in D_{k-1}^{a}$, $d_{k}=a$, and $x_{k+1}^{A}=H$. Then, $x_{\theta}^{A}=x_{\theta+1}^{A}=H$, so $P_{\sigma^{*}}^{A}\left(\bar{d}_{k-1}^{A} \mid s\right)=P(H \mid s)^{2}(\lambda / 2)^{\frac{\theta-1}{2}}$, which implies that

$$
P_{\sigma^{*}}^{A}\left(\bar{d}_{k-1}^{A}, d_{k} \mid s\right)=P(H \mid s)^{2}(\lambda / 2)^{\frac{\theta-1}{2}}\left(1-P(L \mid s)^{3} \Lambda(k-1)\right),
$$

by Lemma C2. Hence,

$$
\phi_{k-1}^{A *}\left(\bar{d}_{k-1}^{A}, d_{k}, H\right)=\frac{p^{3}\left(1-(1-p)^{3} \Lambda(k-1)\right)}{p^{3}\left(1-(1-p)^{3} \Lambda(k-1)\right)+(1-p)^{3}\left(1-p^{3} \Lambda(k-1)\right)} .
$$

Since $p>\frac{1}{2}$, then (i) $p^{3}>(1-p)^{3}$ and (ii) $1-(1-p)^{3} \Lambda(k-1)>1-p^{3} \Lambda(k-1)$. Thus, $\phi_{k-1}^{A *}\left(\bar{d}_{k-1}^{A}, H\right)>\frac{1}{2}$, as shown in Table C3.

For each cell in Table C3, there is a unique sequentially rational action, which we record in Table B2(a).

Observe that if player $k+1^{A}$ observes a history $\bar{d}_{k}^{A}$ that's not given by a row of Table $\mathrm{B} 2(\mathrm{a})$, then $\bar{d}_{k}^{A}$ is not an equilibrium path history, so the belief and strategy of $k+1^{A}$ are arbitrary.

Proof of Lemma B3.2. Without loss we consider player $k+2^{A}$. By Lemmas B1, B2, and B3.1, any equilibrium path history $\left(\bar{d}_{k-1}^{A}, d_{k}, d_{k+1}^{A}\right)$ belongs to one of the rows in Table B2(b). Let $\left(\bar{d}_{k-1}^{A}, d_{k}, d_{k+1}^{A}\right)=\left(d_{1}^{A}, \ldots, d_{k-1}^{A}, d_{k}, d_{k+1}^{A}\right)$ be such a history. If $\bar{d}_{k-1}^{A} \in D_{k-1}^{a}$ or $\bar{d}_{k-1}^{A} \in D_{k-1}^{r}$, then there is an odd integer $j<k-1$ such that $d_{j}^{A}=d_{j+1}^{A}$. Let $\theta$ denote the smallest such $j$. Note that $\left(d_{1}^{A}, \ldots, d_{\theta}^{A}\right)$ is balanced. If $\bar{d}_{k-1}^{A} \in D_{k-1}^{b}$, let $\theta=k-2$. 
We proceed by deriving the belief of player $k+2^{A}$. Recall that

$$
\begin{aligned}
\phi_{k+2}^{A *}\left(\bar{d}_{k-1}^{A}, d_{k}, d_{k+1}^{A}, x_{k+2}^{A}\right) & =\frac{P(\mathcal{H}) P\left(x_{k+2}^{A} \mid \mathcal{H}\right) P_{\sigma^{*}}^{A}\left(\bar{d}_{k-1}^{A}, d_{k}, d_{k+1}^{A} \mid \mathcal{H}\right)}{\sum_{s \in\{\mathcal{H}, \mathcal{L}\}} P(s) P\left(x_{k+2}^{A} \mid s\right) P_{\sigma^{*}}^{A}\left(\bar{d}_{k-1}^{A}, d_{k}, d_{k+1}^{A} \mid s\right)} \\
& =\frac{P(\mathcal{H}) P\left(x_{k+2}^{A} \mid \mathcal{H}\right) P_{\sigma^{*}}^{A}\left(\bar{d}_{k-1}^{A}, d_{k} \mid \mathcal{H}\right) P_{\sigma^{*}}^{A}\left(d_{k+1}^{A} \mid \mathcal{H}, \bar{d}_{k-1}^{A}, d_{k}\right)}{\sum_{s \in\{\mathcal{H}, \mathcal{L}\}} P(s) P\left(x_{k+2}^{A} \mid s\right) P_{\sigma^{*}}^{A}\left(\bar{d}_{k-1}^{A}, d_{k} \mid s\right) P_{\sigma^{*}}^{A}\left(d_{k+1}^{A} \mid s, \bar{d}_{k-1}^{A}, d_{k}\right)} .
\end{aligned}
$$

Lemma B1 implies that players $1^{A}$ to $\theta+1^{A}$ follow their own signals, while players $\theta+2^{A}$ to $k-1^{A}$ are in a cascade. Thus, $x_{i}^{A}=H$ if $d_{i}^{A}=a$ and $x_{i}^{A}=L$ if $d_{i}^{A}=r$ for all $i \in\{1, \ldots, \theta+1\}$. Hence, $P_{\sigma^{*}}^{A}\left(\bar{d}_{k-1}^{A} \mid s\right)=P\left(x_{\theta}^{A} \mid s\right) P\left(x_{\theta+1}^{A} \mid s\right)(\lambda / 2)^{\frac{\theta-1}{2}}$. We use this expression, Lemma C2 and Lemma B3.1 to write the following table that describes when the belief of player $k+2^{A}$ is greater than, equal to, or less than $\frac{1}{2}$.

\begin{tabular}{ccccc} 
& & & \multicolumn{3}{c}{$\phi_{k+2}^{A *}(\cdot)$} \\
\cline { 3 - 5 } $\bar{d}_{k-1}^{g}$ & $d_{k}$ & $d_{k+1}^{g}$ & $x_{k+2}^{A}=H$ & $x_{k+2}^{A}=L$ \\
\hline \multirow{2}{*}{$D_{k-1}^{a}$} & $a$ & $a$ & $\phi_{k+2}^{A *}>\frac{1}{2}$ & $\phi_{k+2}^{A *}>\frac{1}{2}$ \\
\cline { 2 - 5 } & $r$ & $a$ & $\phi_{k+2}^{A *}>\frac{1}{2}$ & $\phi_{k+2}^{A *}<\frac{1}{2}$ \\
\cline { 2 - 5 } & $r$ & $r$ & $\phi_{k+2}^{A *}<\frac{1}{2}$ & $\phi_{k+2}^{A *}<\frac{1}{2}$ \\
\hline$D_{k-1}^{b}$ & $a$ & $a$ & $\phi_{k+2}^{A *}>\frac{1}{2}$ & $\phi_{k+2}^{A *}>\frac{1}{2}$ \\
\cline { 2 - 5 } & $r$ & $r$ & $\phi_{k+2}^{A *}<\frac{1}{2}$ & $\phi_{k+2}^{A *}<\frac{1}{2}$ \\
\hline \multirow{2}{*}{$D_{k-1}^{r}$} & $a$ & $a$ & $\phi_{k+2}^{A *}>\frac{1}{2}$ & $\phi_{k+2}^{A *}>\frac{1}{2}$ \\
\cline { 2 - 5 } & $a$ & $r$ & $\phi_{k+2}^{A *}>\frac{1}{2}$ & $\phi_{k+2}^{A *}<\frac{1}{2}$ \\
\cline { 2 - 5 } & $r$ & $r$ & $\phi_{k+2}^{A *}<\frac{1}{2}$ & $\phi_{k+2}^{A *}<\frac{1}{2}$ \\
\hline
\end{tabular}

Table C4: When $\phi_{k+2}^{A *}(\cdot)$ is $>,=$, and $<1 / 2$

We illustrate the calculation of the left most cell of the second row of Table C4, i.e., when $\bar{d}_{k-1}^{A} \in D_{k-1}^{a}, d_{k}=r, d_{k+1}^{A}=a$, and $x_{k+2}^{A}=H$. Then $x_{\theta}^{A}=x_{\theta+1}^{A}=H$ and Lemma C2 implies

$$
P_{\sigma^{*}}^{A}\left(\bar{d}_{k-1}^{A}, d_{k} \mid s\right)=P(H \mid s)^{2}(\lambda / 2)^{\frac{\theta-1}{2}} P(L \mid s)^{3} \Lambda(k-1) .
$$

Since $k+1^{A}$ follows her signal by Lemma B3.1, $x_{k+1}^{A}=H$. Thus

$$
\phi_{k+2}^{A *}\left(\bar{d}_{k+1}^{A}, r, a, H\right)=\frac{p^{4}(1-p)^{3} \Lambda(k-1)}{p^{4}(1-p)^{3} \Lambda(k-1)+p^{3}(1-p)^{4} \Lambda(k-1)}=p>1 / 2 .
$$

For each cell in Table C4, there is a unique sequentially rational action, which we record in Table B2(b). 
Observe that if player $k+2^{A}$ observes a history $\bar{d}_{k+2}^{A}$ that's not given by a row of Table $\mathrm{B} 2$ (b), then $\bar{d}_{k+2}^{A}$ is not an equilibrium path history, so the belief and strategy of $k+1^{A}$ are arbitrary.

Proof of Lemma B3.3. Without loss we focus on group A. We proceed in two steps. First, we show that every player $i^{A}>k+2^{A}$ is in a cascade on the action of player $k+2^{A}$ when $k+2^{A}$ is herself in a cascade. Second, we show that if player $k+2^{A}$ follows her own signal then the behavior of subsequent players is given by an analog of Lemma B.1, to be made precise below. We conclude by using these two facts to establish Table B2(c).

We use an inductive argument to show that if player $k+2^{A}$ is in a cascade then every subsequent player is also in a cascade on the same action. Lemma B3.2 gives that player $k+2^{A}$ is in a cascade on $a$ if the history she observes $\left(\bar{d}_{k-1}^{A}, d_{k}, d_{k+1}^{A}\right)$ is such that

1. $\bar{d}_{k-1}^{A} \in D_{k-1}^{a}, d_{k}=a$, and $d_{k+1}^{A}=a$,

2. $\bar{d}_{k-1}^{A} \in D_{k-1}^{b}, d_{k}=a$, and $d_{k+1}^{A}=a$, or

3. $\bar{d}_{k-1}^{A} \in D_{k-1}^{r}, d_{k}=a$, and $d_{k+1}^{A}=a$.

Likewise, player $k+2^{A}$ is in a cascade on $r$ if the history she observes $\left(\bar{d}_{k-1}^{A}, d_{k}, d_{k+1}^{A}\right)$ is such that

4. $\bar{d}_{k-1}^{A} \in D_{k-1}^{a}, d_{k}=r$, and $d_{k+1}^{A}=r$,

5. $\bar{d}_{k-1}^{A} \in D_{k-1}^{b}, d_{k}=r$, and $d_{k+1}^{A}=r$, or

6. $\bar{d}_{k-1}^{A} \in D_{k-1}^{r}, d_{k}=r$, and $d_{k+1}^{A}=r$.

Consider player $k+3^{A}$. Since player $k+2^{A}$ is in a cascade for these histories, player $k+3^{A}$ learns nothing from her equilibrium action. Thus, the beliefs of players $k+2^{A}$ and $k+3^{A}$ are the same, provided they each get the same signal realization, i.e., for each $x \in\{H, L\}$,

$$
\phi_{k+2}^{A *}\left(\bar{d}_{k-1}^{A}, d_{k}, d_{k+1}^{A}, x\right)=\phi_{k+3}^{A *}\left(\bar{d}_{k-1}^{A}, d_{k}, d_{k+1}^{A}, d_{k+2}^{A}, x\right),
$$

where $d_{k+1}^{A}=\sigma_{k+1}^{A *}\left(\bar{d}_{k-1}^{A}, d_{k}, d_{k+1}^{A}, x\right)$. Thus, the equilibrium mapping from signals to actions is the same for players $k+2^{A}$ and $k+3^{A}$. Since $k+2^{A}$ is in a cascade, player $k+3^{A}$ is also in a cascade on the same action. 
By the same reasoning, player $k+4^{A}$ learns nothing from the equilibrium actions of players $k+2^{A}$ or $k+3^{A}$, and has the same equilibrium belief and takes the same equilibrium action as these players. By the same logic, every subsequent player is in a cascade on the action of player $k+2^{A}$.

We now show that when player $k+2^{A}$ follows her own signal, i.e., when

1. $\bar{d}_{k-1}^{A} \in D_{k-1}^{a}, d_{k}=r$, and $d_{k+1}^{A}=a$, or

2. $\bar{d}_{k-1}^{A} \in D_{k-1}^{r}, d_{k}=a$, and $d_{k+1}^{A}=r$,

then the behavior of every subsequent player is given by the following table, which is analogous to Lemma B1.

\begin{tabular}{ccc} 
& \multicolumn{2}{c}{$\sigma_{i}^{A *}\left(\cdot, d_{k+2}^{A}, \ldots, d_{i-1}^{A}, x_{i}^{A}\right)$} \\
\cline { 2 - 3 }$\left(d_{k+2}^{A}, \ldots, d_{i-1}^{A}\right)$ & $H$ & $L$ \\
\hline$D_{i-k-2}^{a}$ & $a$ & $a$ \\
\hline$D_{i-k-2}^{b}$ & $\mathbf{a}$ & $\mathbf{r}$ \\
\hline$D_{i-k-2}^{r}$ & $r$ & $r$ \\
\hline
\end{tabular}

Table entries in bold indicate when a player follows her signal.

Table C5: Equilibrium Play of Players $i^{A}>k+2^{A}$ When $k+2^{A}$ Follows Her Signal Table C5 gives that player $i^{A}$ 's equilibrium strategy depends only on the decisions of players $k+2^{A}$ through $i-1^{A}$ and her own signal.

We begin by noting that

$$
P_{\sigma^{*}}^{A}\left(\bar{d}_{k-1}^{A}, d_{k}, d_{k+1}^{A} \mid \mathcal{H}\right)=P_{\sigma^{*}}^{A}\left(\bar{d}_{k-1}^{A}, d_{k}, d_{k+1}^{A} \mid \mathcal{L}\right) .
$$

For concreteness, suppose case (1). By equation (3) of the Proof of Lemma B2.2, we have that

$$
P_{\sigma^{*}}^{A}\left(\bar{d}_{k-1}^{A}, d_{k}, d_{k+1}^{A} \mid s\right)=P(H \mid s)^{3}(\lambda / 2)^{\frac{\theta-1}{2}} P(L \mid s)^{3} \Lambda(k-1) .
$$

Since $P(H \mid s)^{3} P(L \mid s)^{3}=p^{3}(1-p)^{3}$ for all states $s$ and since $\lambda$ and $\Lambda$ are independent of $s$, equation (4) holds; the argument for case (2) is analogous. 
Consider player $k+3^{A}$. Her belief is

$$
\begin{aligned}
& \phi_{k+3}^{A *}\left(\bar{d}_{k-1}^{A}, d_{k}, d_{k+1}^{A}, d_{k+2}^{A}, x_{k+3}^{A}\right) \\
& =\frac{P(\mathcal{H}) P\left(x_{k+3}^{A} \mid \mathcal{H}\right) P_{\sigma^{*}}^{A}\left(\bar{d}_{k-1}^{A}, d_{k}, d_{k+1}^{A} \mid \mathcal{H}\right) P_{\sigma^{*}}^{A}\left(d_{k+2}^{A} \mid \mathcal{H}, \bar{d}_{k-1}^{A}, d_{k}, d_{k+1}^{A}\right)}{\sum_{s \in\{\mathcal{H}, \mathcal{L}\}} P(s) P\left(x_{k+3}^{A} \mid s\right) P_{\sigma^{*}}^{A}\left(\bar{d}_{k-1}^{A}, d_{k}, d_{k+1}^{A} \mid s\right) P_{\sigma^{*}}^{A}\left(d_{k+2}^{A} \mid s, \bar{d}_{k-1}^{A}, d_{k}, d_{k+1}^{A}\right)} \\
& =\frac{P(\mathcal{H}) P\left(x_{k+3}^{A} \mid \mathcal{H}\right) P_{\sigma^{*}}^{A}\left(d_{k+2}^{A} \mid \mathcal{H}, \bar{d}_{k-1}^{A}, d_{k}, d_{k+1}^{A}\right)}{\sum_{s \in\{\mathcal{H}, \mathcal{L}\}} P(s) P\left(x_{k+3}^{A} \mid s\right) P_{\sigma^{*}}^{A}\left(d_{k+2}^{A} \mid s, \bar{d}_{k-1}^{A}, d_{k}, d_{k+1}^{A}\right)},
\end{aligned}
$$

where the third line follows from equation (4). Lemma B3.2 gives that player $k+2^{A}$ follows her own signal, so $P_{\sigma^{*}}^{A}\left(d_{k+2}^{A} \mid \mathcal{H}, \bar{d}_{k-1}^{A}, d_{k}, d_{k+1}^{A}\right)=P\left(x_{k+2}^{A} \mid s\right)$ for each state $s$, where $x_{k+2}^{A}=H$ if $d_{k+2}^{A}=a$ and $x_{k+2}^{A}=L$ if $d_{k+2}^{A}=r$. Thus,

$$
\phi_{k+3}^{A *}\left(\bar{d}_{k-1}^{A}, d_{k}, d_{k+1}^{A}, d_{k+2}^{A}, x_{k+3}^{A}\right)=\frac{P(\mathcal{H}) P\left(x_{k+3}^{A} \mid \mathcal{H}\right) P\left(x_{k+2}^{A} \mid \mathcal{H}\right)}{\sum_{s \in\{\mathcal{H}, \mathcal{L}\}} P(s) P\left(x_{k+3}^{A} \mid s\right) P\left(x_{k+2}^{A} \mid s\right)} .
$$

Since (i) $d_{k+2}^{A} \in D_{k+3-k-2}^{b}$ and, as in the Proof of Lemma B1, (ii) $\phi_{k+3}^{A *}(\cdot, H) \geq 1 / 2$ and $\phi_{k+3}^{A *}(\cdot, L) \leq 1 / 2$ for all $d_{k+2}^{A}$, Table C5 describes the equilibrium action of player $k+3^{A}$.

Suppose Table C5 describes the equilibrium behavior of players $k+3^{A}$ to $i-1^{A}$. We show that Table C5 also describes the behavior of player $i^{A}$. Write

$$
P_{\sigma^{*}}^{A}\left(\bar{d}_{k-1}^{A}, d_{k}, d_{k+1}^{A}, \ldots, d_{i-1}^{A} \mid s\right)=P_{\sigma^{*}}^{A}\left(\bar{d}_{k-1}^{A}, d_{k}, d_{k+1}^{A} \mid s\right) P_{\sigma^{*}}^{A}\left(d_{k+2}^{A}, \ldots, d_{i-1}^{A} \mid s, \bar{d}_{k-1}^{A}, d_{k}, d_{k+1}^{A}\right)
$$

There are three cases by the induction hypothesis, (i) $\left(d_{k+2}^{A}, \ldots, d_{i-1}^{A}\right) \in D_{i-k-2}^{b}$,

(ii) $\left(d_{k+2}^{A}, \ldots, d_{i-1}^{A}\right) \in D_{i-k-2}^{a}$, and (iii) $\left(d_{k+2}^{A}, \ldots, d_{i-1}^{A}\right) \in D_{i-k-2}^{a}$.

Case (i): If $\left(d_{k+2}^{A}, \ldots, d_{i-1}^{A}\right) \in D_{i-k-2}^{b}$, then the induction hypothesis implies that each player $k+2^{A}$ to $i-1^{A}$ has followed her own signal. Thus,

$$
P_{\sigma^{*}}^{A}\left(d_{k+2}^{A}, \ldots, d_{i-1}^{A} \mid s, \bar{d}_{k-1}^{A}, d_{k}, d_{k+1}^{A}\right)=\prod_{j=k+2}^{i-1} P\left(x_{j}^{A} \mid s\right) \text { where } x_{j}^{A}=\left\{\begin{array}{cl}
H & \text { if } d_{j}^{A}=a \\
L & \text { if } d_{j}^{A}=r
\end{array}\right.
$$

If $i$ is odd then $P_{\sigma^{*}}^{A}\left(d_{k+2}^{A}, \ldots, d_{i-1}^{A} \mid s, \bar{d}_{k-1}^{A}, d_{k}, d_{k+1}^{A}\right)=(\lambda / 2)^{\frac{i-k-2}{2}}$, and if $i$ is even then $P_{\sigma^{*}}^{A}\left(d_{k+2}^{A}, \ldots, d_{i-1}^{A} \mid s, \bar{d}_{k-1}^{A}, d_{k}, d_{k+1}^{A}\right)=(\lambda / 2)^{\frac{i-k-3}{2}} P\left(x_{i-1}^{A} \mid s\right)$. Thus player $i$ 's belief given signal 
$x_{i}^{A}$ is

$$
\begin{aligned}
& \phi_{i}^{A *}\left(\bar{d}_{k-1}^{A}, d_{k}, d_{k+1}^{A}, d_{k+2}^{A}, \ldots, d_{i-1}^{A}, x_{i}^{A}\right) \\
& =\frac{P(\mathcal{H}) P\left(x_{i}^{A} \mid \mathcal{H}\right) P_{\sigma^{*}}^{A}\left(\bar{d}_{k-1}^{A}, d_{k}, d_{k+1}^{A}, \ldots, d_{i-1}^{A} \mid \mathcal{H}\right)}{\sum_{s \in\{\mathcal{H}, \mathcal{L}\}} P(s) P\left(x_{i}^{A} \mid s\right) P_{\sigma^{*}}^{A}\left(\bar{d}_{k-1}^{A}, d_{k}, d_{k+1}^{A}, \ldots, d_{i-1}^{A} \mid s\right)} \\
& =\frac{P(\mathcal{H}) P\left(x_{i}^{A} \mid \mathcal{H}\right) P_{\sigma^{*}}^{A}\left(d_{k+2}^{A}, \ldots, d_{i-1}^{A} \mid \mathcal{H}, \bar{d}_{k-1}^{A}, d_{k}, d_{k+1}^{A}\right)}{\sum_{s \in\{\mathcal{H}, \mathcal{L}\}} P(s) P\left(x_{i}^{A} \mid s\right) P_{\sigma^{*}}^{A}\left(d_{k+2}^{A}, \ldots, d_{i-1}^{A} \mid s, \bar{d}_{k-1}^{A}, d_{k}, d_{k+1}^{A}\right)} \\
& =\left\{\begin{array}{cc}
\frac{P(\mathcal{H}) P\left(x_{i}^{A} \mid \mathcal{H}\right)}{\sum_{s \in\{\mathcal{H}, \mathcal{L}\}} P(s) P\left(x_{i} \mid s\right)} & \text { if } i \text { is odd. } \\
\frac{P(\mathcal{H}) P\left(x_{i}^{A} \mid \mathcal{H}\right) P\left(x_{i-1}^{A} \mid \mathcal{H}\right)}{\sum_{s \in\{\mathcal{H}, \mathcal{L}\}} P(s) P\left(x_{i}^{A} \mid s\right) P\left(x_{i-1}^{A} \mid s\right)} & \text { if } i \text { is even. }
\end{array}\right.
\end{aligned}
$$

As in the proof of Lemma B1, $\phi_{i}^{A *}(\cdot, H) \geq 1 / 2$ for all $d_{i-1}^{A}$ and $\phi_{i}^{*}(\cdot, L) \leq 1 / 2$ for all $\bar{d}_{i-1}^{A}$, so player $i^{A}$ follows her own signal as in Table C5.

Case (ii): If $\left(d_{k+2}^{A}, \ldots, d_{i-1}^{A}\right) \in D_{i-k-2}^{a}$, then there is an odd integer $j$ between $k+2$ and $i-1$ such that $d_{j}^{A}=d_{j+1}^{A}=a$. Let $\theta$ denote the smallest such $j$. Note that $\left(d_{k+2}^{A}, \ldots, d_{\theta}^{A}\right)$ is balanced. By the induction hypothesis (a) players $k+2^{A}$ through $\theta+1^{A}$ follow their own signals and (b) players $\theta+2^{A}$ through $i-1^{A}$ take the same action as player $\theta+1^{A}$ regardless of their own signals. Thus

$$
P_{\sigma^{*}}^{A}\left(d_{k+2}^{A}, \ldots, d_{i-1}^{A} \mid s, \bar{d}_{k-1}^{A}, d_{k}, d_{k+1}^{A}\right)=\prod_{j=k+2}^{\theta+1} P\left(x_{j}^{A} \mid s\right) \text { where } x_{j}^{A}=\left\{\begin{array}{cl}
H & \text { if } d_{j}^{A}=a \\
L & \text { if } d_{j}^{A}=r
\end{array}\right.
$$

Since $\left(d_{k+2}^{A}, \ldots, d_{\theta}^{A}\right)$ is balanced and $d_{\theta}^{A}=d_{\theta+1}^{A}=a$ then

$$
P_{\sigma^{*}}^{A}\left(d_{k+2}^{A}, \ldots, d_{i-1}^{A} \mid s, \bar{d}_{k-1}^{A}, d_{k}, d_{k+1}^{A}\right)=(\lambda / 2)^{\frac{\theta-k-2}{2}} P\left(x_{\theta}^{A} \mid s\right) P\left(x_{\theta+1}^{A} \mid s\right)=(\lambda / 2)^{\frac{\theta-k-2}{2}} P(H \mid s)^{2} .
$$

Hence

$$
\phi_{i}^{A *}\left(\bar{d}_{k-1}^{A}, d_{k}, d_{k+1}^{A}, d_{k+2}^{A}, \ldots, d_{i-1}^{A}, x_{i}^{A}\right)=\frac{P(\mathcal{H}) P\left(x_{i}^{A} \mid \mathcal{H}\right) P(H \mid \mathcal{H})^{2}}{\sum_{s \in\{\mathcal{H}, \mathcal{L}\}} P(s) P\left(x_{i}^{A} \mid s\right) P(H \mid s)^{2}}
$$

It is readily verified that $\phi_{i}^{A *}\left(\cdot, x_{i}\right)>1 / 2$ for $x_{i} \in\{H, L\}$. Thus sequential rationality implies $\sigma_{i}^{*}\left(\cdot, x_{i}\right)=a$, as in Table C5.

Case (iii): The proof is symmetric to Case (ii) and therefore omitted.

Table B2(c) follows directly from the two results we just established. Lemmas B1, B2, and B3.1 give that player $i^{A}$ observes, in equilibrium, a history $\left(\bar{d}_{k-1}^{A}, d_{k}, d_{k+1}^{A}, d_{k+2}^{A}, \ldots, d_{i-1}^{A}\right)$ where $\left(\bar{d}_{k-1}^{A}, d_{k}, d_{k+1}^{A}\right)$ is from a row in Table B2(c). If $\left(\bar{d}_{k-1}^{A}, d_{k}, d_{k+1}^{A}\right)$ is such that player $k+2^{A}$ is in a cascade, then our first result pins down the equilibrium 
decisions of all subsequent player. Thus, we obtain rows 1, 5, 6, 7, 8, and 12 of Table B2(c). While if $\left(\bar{d}_{k-1}^{A}, d_{k}, d_{k+1}^{A}\right)$ is such that player $k+2^{A}$ is not in a cascade, then Table C5 pins down the equilibrium decisions of all subsequent players. Thus, we obtain rows $2,3,4,9,10$, and 11 of Table B2(c).

Observe that if player $i^{A}$ observes a history $\bar{d}_{i-1}^{A}$ that's not given by a row of Table B2(b), then $\bar{d}_{i-1}^{A}$ is not an equilibrium path history, so the belief and strategy of $i^{A}$ are arbitrary. 